\title{
Quantum kinetics of anomalous and nonlinear Hall effects in topological semimetals
}

\author{
Elio J. König ${ }^{\mathrm{a}}$, Alex Levchenko ${ }^{\mathrm{b}}$ \\ ${ }^{a}$ Max Planck Institute for Solid State Research, Heisenbergstrasse 1, D-70569 Stuttgart, Germany \\ ${ }^{b}$ Department of Physics, University of Wisconsin-Madison, Madison, Wisconsin 53706, USA
}

\begin{abstract}
We present a systematic microscopic derivation of the semiclassical Boltzmann equation for band structures with the finite Berry curvature based on Keldysh technique of nonequilibrium systems. In the analysis, an ac electrical driving field is kept up to quadratic order, and both cases of small and large frequencies corresponding to intra- and interband transitions are considered. In particular, this formulation is suitable for the study of nonlinear Hall effect and photogalvanic phenomena. The role of impurity scattering is carefully addressed. Specifically, in addition to previously studied side-jump and skew-scattering processes, quantum interference diffractive contributions are now explicitly incorporated within the developed framework. This theory is applied to multifold fermions in topological semimetals, for which the generic formula for the skew scattering rate from the Pancharatnam phase is obtained along with the corresponding anomalous Hall conductivity.
\end{abstract}

Keywords: Anomalous Hall effect, Berry curvature, skew scattering, side jump, Weyl nodes

\section{Contents}

1 Introduction $\quad 2$

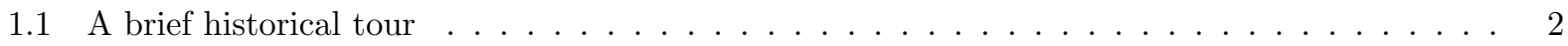

1.2 Technical synopsis of previous works $\ldots \ldots \ldots \ldots \ldots \ldots \ldots \ldots \ldots$

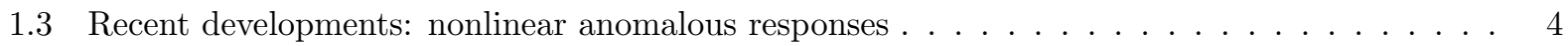

1.4 Overview of this work $\ldots \ldots \ldots \ldots \ldots \ldots \ldots \ldots$

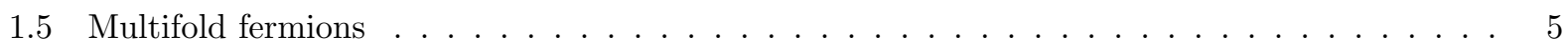

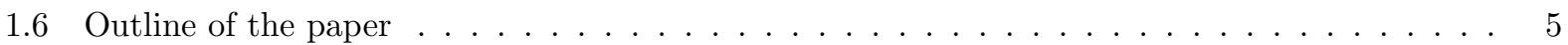

2 Derivation of quantum kinetic equation $\quad 5$

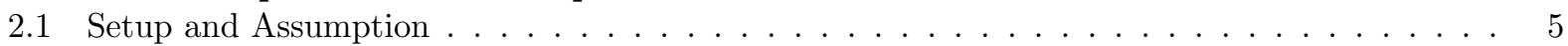

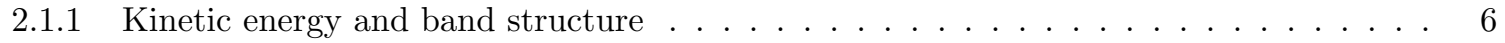

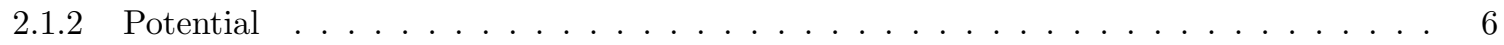

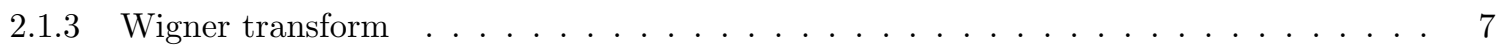

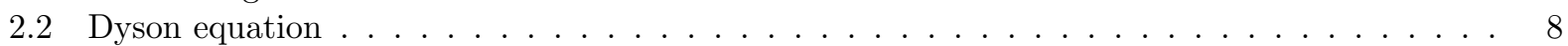

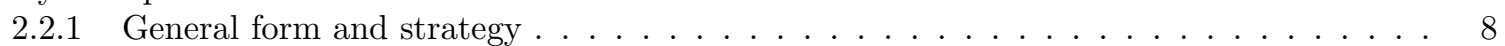

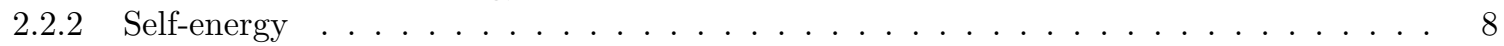

2.2 .3 Interband Keldysh function $\ldots \ldots \ldots \ldots \ldots \ldots$

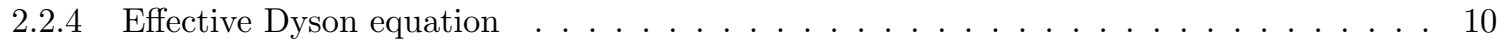

2.3 Semiclassical expansion for $\omega \ll E_{F} \quad \ldots \ldots \ldots \ldots \ldots \ldots \ldots \ldots$

2.3.1 Liouville term . . . . . . . . . . . . . . . . . . . . . . . . . 10

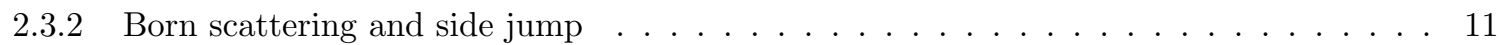

2.3 .3 Skew scattering . . . . . . . . . . . . . . . . . . . . . . . . . 11

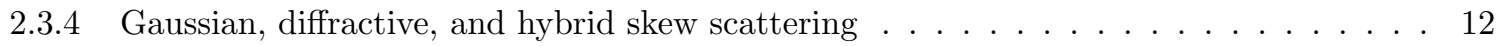

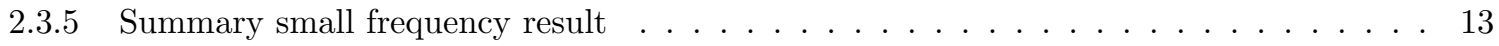

Preprint submitted to Annals of Physics: contribution to the Philip W. Anderson Memorial Special Issue April 23, 2021 
2.4 Semiclassical expansion for $\omega \gtrsim E_{F} \ldots \ldots \ldots \ldots \ldots \ldots$

2.4.1 Liouville term and disorder scattering . . . . . . . . . . . . . . . . . . . . . . . . . . . . . . . . . .

2.4 .2 Injection and shift currents . . . . . . . . . . . . . . . . . . . . 14

2.4.3 Mixed disorder and photon scattering . . . . . . . . . . . . . . . . . . 14

2.4.4 Summary of large frequency result . . . . . . . . . . . . . . . . . . . . . 14

3 Electrical current response

4 Anomalous Hall transport of multifold fermions $\quad 15$

4.1 Eigenstates and Berry curvature . . . . . . . . . . . . . . . . . . 16

4.2 Projectors on eigenstates . . . . . . . . . . . . . . . . . . . . . . 16

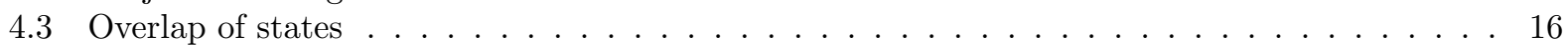

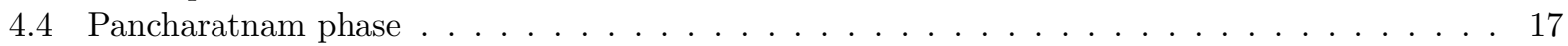

4.5 AHE of pseudospin-1 fermions . . . . . . . . . . . . . . . . . 18

5 Summary and Outlook 19

6 Acknowledgments $\quad 19$

$\begin{array}{llll}\text { Appendix A } & \text { Collision integrals and averages } & 19\end{array}$

\section{Introduction}

\subsection{A brief historical tour}

The anomalous Hall effect (AHE), including its spin, valley, thermoelectric, and quantized counterparts, in their complexity represent perhaps the most fascinating set of quantum transport phenomena, see Refs. [16 ] for reviews and references herein. At the same time, anomalous transport coefficients provide key insights to correlated and topological quantum materials. This includes the intriguing normal state of cuprate superconductors, with an anomalous temperature scaling of Hall and longitudinal resistivity which Anderson traced back to his theory of tomographic Luttinger liquids [7, 8], as well as a giant enhancement of the thermal Hall effect [9]. In the presence of spin-orbit coupling, a large anomalous Hall effect also gives access to the physics of local moments, first discussed by Kondo [10] and later extended to the mixed valence regime by Coleman, Anderson and Ramakrishnan [11, 12], who demonstrate a massively enhanced skew scattering off quantum impurities. Finally, Kerr rotation experiments [13], which probe the finite frequency anomalous Hall conductance, have become one of two litmus tests for time reversal symmetry breaking superconductors, i.e. solid state cousins of unconventional pairing states pioneered by Anderson, Brinkman and Morel in the context of ${ }^{3} \mathrm{He}[14,15]$ (the other litmus test being muon-spin-rotation).

In retrospect, when counting from the experimental discovery by Edwin Hall in 1880 it took seven decades for a comprehensive theory of the AHE to be developed even for weakly coupled materials. In their seminal work Karplus and Luttinger [16] laid out foundations of the AHE by elaborating rigorous microscopic linear-response calculations in multi-band metals, recognizing importance of spin-orbit interaction, and most importantly discovering an anomalous group velocity term in the semiclassical equation of motion of Bloch electrons in solids. During the following several decades the extrinsic mechanisms of anomalous Hall transport where uncovered and scrutinized. Smit [17, 18] found a skew scattering mechanism of AHE that captures left-right asymmetry in the differential scattering cross section of a conduction electron on an impurity potential. Berger [19] studied another subtle quantum side-jump contribution, which occurs due to coordinate shifts in electron trajectories following the consecutive scattering events.

The spin version of the Hall effect was proposed by Dyakonov and Perel [20, 21], which consists of spin accumulation by passing an electrical current. This insightful work ultimately triggered another cycle of numerous studies where intrinsic and extrinsic origins of the spin Hall effect (SHE) were debated.

The discovery of the integer Hall effect by von Klitzing, Dorda, and Pepper [22] and its further explanation eventually greatly contributed to the deeper understanding of the AHE through the work of Thouless, 
Kohmoto, Nightingale, and den Nijs (TKNN) [23] who derived their famous invariant for the Hall conductance formula. The next crucial steps was done by Haldane [24] who presented a concrete model for the quantized anomalous Hall effect (QAHE) in a lattice system. Shortly after that, the geometric and topological aspects of QAHE were understood and quickly became the dominant paradigm. The anomalous velocity was connected to the Berry phase [25] so it became possible to express the anomalous Hall conductance in terms of the integral of the Berry curvature over the momentum space [26]. This ultimately gave a representation of quantization in terms of the Chern number of fully filled bands.

These advances elucidated the geometrical origin of the AHE even in the situations when it is not quantized. This is in fact true not only for the intrinsic mechanism, but also applicable to extrinsic mechanisms: skew scattering amplitudes as well since coordinate shifts in the side-jump processes can be expressed in terms of Pancharatnam phase [27], which represents a special case of Berry phase. Indeed, gauge invariant formulae for coordinate displacements of electrons undergoing quantum transitions were known early on from work of Belinicher, Ivchenko, and Sturman [28] on the kinetic theory of photovoltaic effect, however, the topological aspects of the problem were not realized at that time. This work was rediscovered only relatively recently and provided renewed important insights [29]. The peculiar observation is that at least in some models of the disorder potential the microscopic characteristics of the impurity potential drop out from the final expression for the coordinate shifts that thus can be expressed only in terms of electronic Bloch functions. This feature makes certain extrinsic contributions to some extent universal.

The quantum version of the spin Hall effect (QSHE) was discussed by Kane and Mele [30] in the context of a hexagonal lattice model as in graphene. The description was constructed from the generalized model of Haldane extended to spin- $\frac{1}{2}$ electrons with spin-orbit coupling. However, weak spin-orbit interaction in graphene stimulated further searches of material platforms where this effect could manifest. The CdTe/HgTe heterostructure was proposed by Bernevig, Hughes, and Zhang [31] to have the right ingredients with an inverted band structure for the proper thickness of quantum wells when a topological phase transition occurs. This prediction was confirmed experimentally [32] via observed robust conductance quantization facilited by topologically protected edge modes. The time reversal broken analog, i.e. the quantum anomalous Hall effect was recently observed in thin films of magnetic topological insulators [33] as well as quantum valley Hall effect in graphene superlattices [34]. More generally, graphene bilayers at magic twist angle provide the most recent experimental platform for the study of interaction driven emergent ferromagnetic state exhibiting giant anomalous Hall response [35] and possibly topological Chern insulating behavior.

From the present day perspective, it is perhaps fair to say that it took the physics community a whole century to fully understand and conceptualize the plethora of anomalous Hall effects. This is certainly a noble achievement, but in hindsight one is left with a sobering realization that all that intellectual advance concerns essentially a single particle physics in the linear response transport problem. To a large extent we still know very little about effects of interactions [36-41], quantum interference corrections [42-45], and strong nonequilibrium conditions on linear and nonlinear anomalous Hall responses. In part this motivates our work to advance current theory further and bridge the gap towards modeling of experiments.

\subsection{Technical synopsis of previous works}

At the technical level there exist several distinct approaches to describe generalities of the anomalous Hall effect. An analytical theory based on a transport like equation for the density matrix was developed by Luttinger [46]. In this framework effects of impurities can be incorporated perturbatively by a systematic expansion in disorder potential. This treatment provides rigorous and controllable quantum-mechanical description which also reveals importance of the off-diagonal elements of the density matrix and of the velocity operator in the calculation of conductivity tensor. However, this method is not very practical and difficult to implement even in relatively simple model cases.

A precursor to modern semiclassical theory is the work by Adams and Blount [47, 48] who used the picture of particle wave-packet dynamics in a crystal field described by noncommuting coordinates. In a series of applications this formulation was generalized to degenerate bands in the context of group III-V ntype semiconductors [49-51], which initially had difficulties with incorporation of extrinsic contributions. A modern version of these theories can be presented in the form of Eilenberger-type equations for the reduced 
Green's function, which incorporates both short-range impurity scattering, non-Abelian Berry curvature terms stemming from the band degeneracies, as well as quantum anomalies [52-56].

A fully semiclassical description based on the Boltzmann equation, a diagrammatic method based on the Kubo-Streda formulas, as well as Keldysh technique, were applied to the problem of AHE, see Ref. [57] for an overview. It should be stressed that establishing a connection between these methods is not merely a trivial exercise and requires quite laborious calculations [58, 59]. For instance, at the level of the Boltzmann equation, one finds an additional contribution to transverse conductivity termed as an anomalous distribution. Indeed, the emergent asymmetry of the distribution function, even without an asymmetry in the kernel of collision integral, is the result of the side-jump process in the presence of external electric field which modifies energy conservation. The corresponding correction to the distribution function combined with the conventional part of the band velocity leads to the additional Hall current. This term is not immediately evident at the level of diagrammatic Green's function calculation [60] as it is absorbed into the part of sidejump contribution. This also suggests that clear separation of various terms is somewhat ambiguous. At the same time, diagrammatics produces new terms that were missed in all previous approaches. These are hybrid [59] and diffractive [61-63] skew scatterings. The former one is inversely proportional to the impurity concentration, thus resembling the usual skew scattering from non-Gaussian disorder, but it is independent of impurity strength, resembling the side-jump mechanism. The diffractive process is present already at the level of Gaussian disorder and, perhaps counterintuitively, is independent of both impurity concentration and impurity strength due to subtle cancellations, so it scales as the intrinsic term. The validity of these results has been established in different models $[64,65,63,66]$.

\subsection{Recent developments: nonlinear anomalous responses}

The first systematic attempt to extend the semiclassical theory of AHE to the domain of nonlinear Hall responses was presented in the paper by Deyo et al. [67]. The emphasis of the study was put on the linear and circular photogalvanic phenomena, including calculation of the corresponding response tensors in the presence of weak static magnetic field. The analysis was carried out for the bulk crystal symmetries $T_{d}$ and $C_{6 v}$, and the point symmetry $C_{s}$ of a quantum-well structures. Up to that point such calculations were rigorously established only in the clean limit of semiconductor structures [68]. In part motivated by experiments $[69,70]$ further extensions of the theory and applications were tailored towards circular photogalvanic effect in Weyl semimetals [71-74], which can be considered as an ac non-linear Hall response at optical frequencies.

In a parallel line of developments, Sodemann and $\mathrm{Fu}$ [75] demonstrated the topological origin of the transverse Hall-like currents that occur in second-order response to an external electric field. Arguments were put forward that these effects can occur in a wide class of two- and three-dimensional time-reversal invariant and inversion breaking materials, including topological crystalline insulators, transition metal dichalcogenides and Weyl semimetals [76]. The crucial distinction from the linear AHE is that these nonlinear response functions are governed by the dipole moment of the Berry curvature in momentum space. Furthermore, the Berry curvature dipole emerges both in the dc current and also in the second harmonic. These initial results were extended to include effects of skew-scattering and side-jump on the second order responses [77-81].

\subsection{Overview of this work}

In this work we construct kinetic theory of quantum transport in multiband materials with nontrivial band topology. We base our analysis on Keldysh technique for nonequilibrium systems. In the analysis, external potentials are kept to quadratic order which enables us to address nonlinear anomalous transport effects. This includes both intraband processes at low frequency and interband photogalvanic responses. A careful attention is paid to impurity scattering effects as we retain in calculations disorder potential up to the fourth order. This automatically includes all known skew scattering and side jump disorderinduced contributions, and also less studied quantum interference processes. Even though we employ formal machinery to derive kinetic equation, we use semiclassical language and interpretation of emergent terms. For instance at the level of the Dyson equation for self-energies we make a connection between diagrammatic 
and semiclassical approaches. To avoid spurious difficulties at the intermediate steps, we use fully gaugeinvariant construction and incorporate Berry connection explicitly in the Wigner transform of operators. Given already quite a laborious task, we do not consider effects of external magnetic field.

\subsection{Multifold fermions}

As an application of our theory, in Sec. 4 we present the first microscopic study of anomalous impurity scattering in models of multifold fermions, which are generalizations of 2D and 3D Weyl and Dirac semimetals [82-86] that contain $(2 S+1)$-fold degenerate touching point. We also use the terminology of multifold fermions, when the degeneracy at touching point is lifted (in the simplest case, this lifting occurs by a mass gap). The kinetic part of the Hamiltonian in the simplest $\boldsymbol{k} \cdot \boldsymbol{p}$ expansion, is of the form

$$
H_{\text {kin }}(\boldsymbol{p})=d_{0}(\boldsymbol{p})+\sum_{i=1}^{3} d_{i}(\boldsymbol{p}) S_{i} .
$$

Here, $d_{0,1,2,3}(\boldsymbol{p})$ are momentum dependent functions and $S_{i}$ are spin- $S$ matrices, with the usual commutation algebra $\left[S_{i}, S_{j}\right]=i \varepsilon_{i j k} S_{k}$, where $\varepsilon_{i j k}$ is the Levi-Civita symbol. The Hamiltonian (1.1) thereby generalizes the familiar $S=1 / 2$ case applicable, e.g., to Weyl semimetals in 3D and gapped topological insulator surface states in 2D. Particularly 3D multifold fermions [87] have been of great interest for anomalous transport and optics [88-93], which is related to the fact that $\mathrm{RhSi}$ and CoSi contain 4- and 6-fold fermionic touching points [94] and display signatures of quantized photocurrent generation [71, 73, 95]. At the same time, 2D multifold fermions may appear as topological surface states [96] or in appropriately designed lattice models [97-103].

In our study we present general results for $S=1$ and $S=3 / 2$, and evaluate the impurity scattering effects in the case of $S=1$ fermions in detail. We contrast these results to the most well studied $S=1 / 2$ situation in the model of massive 2D Dirac fermions.

\subsection{Outline of the paper}

The rest of the paper is organized as follows. In Sec. 2 the general setup is introduced, along with assumptions and notations. After defining the modified Wigner transform and Moyal expansion, the Dyson equation for the Keldysh block of the Green's function is discussed in details. This block has a non-diagonal structure in the band index and we outline the systematic solution strategy for the off-diagonal quantum components to arrive at kinetic equations for the band-diagonal distribution functions that have natural semiclassical interpretation. These equations are further simplified by following the usual gradient expansion. From the projections onto corresponding bands the collision terms are derived for various processes and topological terms are identified. In the next Sec. 3 the electrical current operator is considered and its semiclassical form is deduced with the side-jump contribution made explicit. These two sections represent the core of the paper. Finally, as mentioned, we present an application to multifold fermions in Sec. 4 and conclude with a summary and outlook in Sec. 5. Some additional technicalities concerning collision kernels and momentum averages are presented in the appendix.

\section{Derivation of quantum kinetic equation}

In this section we present a systematic derivation of the quantum kinetic equation in Berry curved matter in the presence of impurity scattering and external nonlinear drive.

\subsection{Setup and Assumption}

We consider generic $N$-band Hamiltonian containing kinetic and potential terms

$$
H=H_{\text {kin }}(\boldsymbol{p})+U(t, \boldsymbol{x}) .
$$

We now discuss each of these two terms in detail. 


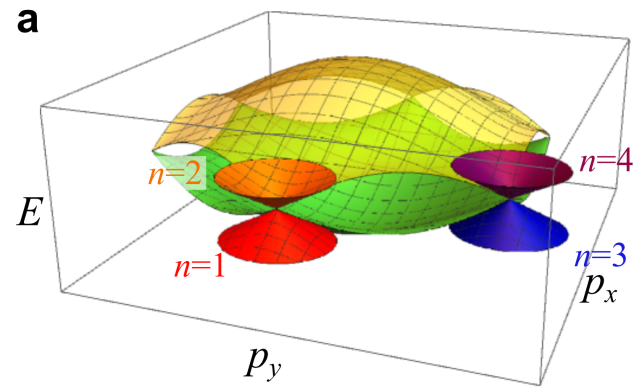

b

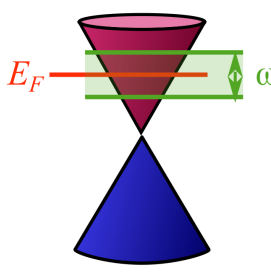

(small frequency)
C

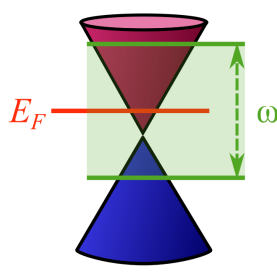

(large frequency)

Figure 1: Illustration of the convention. a We use an $N$-band model (bands are labelled $n=1, \ldots, N$ ) based on the $\boldsymbol{k} \cdot \boldsymbol{p}$ expansion about special points (denoted by $\boldsymbol{b}_{n}$ ) in the Brillouin zone. Here, we exemplify this for the tight binding model on a hexagonal lattice, where we keep only the states near $K$ and $K^{\prime}$ nodes and thus $N=4, \boldsymbol{b}_{1}=\boldsymbol{b}_{2}=(4 \pi / 3,0) / a$ and $\boldsymbol{b}_{3}=\boldsymbol{b}_{4}=(2 \pi / 3,2 \pi / \sqrt{3}) / a$. b (c) Illustration of the possible small (large) frequency regime $\omega \ll E_{F}\left(\omega \gtrsim E_{F}\right)$.

\subsubsection{Kinetic energy and band structure}

We use an effective description of $H_{\text {kin }}(\boldsymbol{p})$ by means of $\boldsymbol{k} \cdot \boldsymbol{p}$ Hamiltonians centered at $N_{c}$ (avoided) nodes in the Brillouin zone denoted $\boldsymbol{b}_{n}$, see Fig. 1 for an illustration with $N=4$ and $N_{c}=2$ (note that $\boldsymbol{b}_{1}=\boldsymbol{b}_{2}$ and $\boldsymbol{b}_{3}=\boldsymbol{b}_{4}$ in this case). The solutions of the free Schrödinger equation are denoted as

$$
\psi_{n, \boldsymbol{p}}(\boldsymbol{x})=\left\langle\boldsymbol{x} \mid \psi_{n, \boldsymbol{p}}\right\rangle=e^{i\left(\boldsymbol{p}-\boldsymbol{b}_{n}\right) \cdot \boldsymbol{x}}\left|u_{n, \boldsymbol{p}}\right\rangle,
$$

and we use the index $n=1, \ldots N$ to label the bands.

The $N$-vectors $\left|u_{n, p}\right\rangle$ constitute the sections of a fibre bundle over momentum space. In what follows we use the usual orthonormality and completeness relations $\left\langle u_{n, \boldsymbol{p}} \mid u_{n^{\prime}, \boldsymbol{p}}\right\rangle=\delta_{n, n^{\prime}}$ and $\sum_{n}\left|u_{n, \boldsymbol{p}}\right\rangle\left\langle u_{n, \boldsymbol{p}}\right|=\mathbf{1}_{N}$. The gauge transformation $\left|u_{n, \boldsymbol{p}}\right\rangle \rightarrow e^{i \phi_{n}(\boldsymbol{p})}\left|u_{n, \boldsymbol{p}}\right\rangle$ is implied by the equivalence of solutions for local (in momentum space), $n$ dependent phase. We consider nondegenerate bands and define the Berry connection as

$$
\mathcal{A}_{n n^{\prime}}(\boldsymbol{p})=i\left\langle u_{n, p}|E| \nabla_{\boldsymbol{p}} u_{n^{\prime}, \boldsymbol{p}}\right\rangle,
$$

which is gauge covariant

$$
\mathcal{A}_{n n^{\prime}} \rightarrow e^{-i \phi_{n}(\boldsymbol{p})}\left[\mathcal{A}_{n n^{\prime}}-\nabla_{\boldsymbol{p}} \phi_{n} \delta_{n, n^{\prime}}\right] e^{i \phi_{n^{\prime}}(\boldsymbol{p})} .
$$

We have introduced the $N \times N$ matrix $E$ which is particularly simple if the dimension of the Hilbert space is equal at each of the $N_{c}$ nodes. Then it can be represented as a block matrix, within each block (diagonal blocks correspond to a given node) it is the $N / N_{c}$-dimensional identity,

$$
E=\left(\begin{array}{ccc}
\mathbf{1}_{N / N_{c}} & \cdots & \mathbf{1}_{N / N_{c}} \\
\vdots & \ddots & \vdots \\
\mathbf{1}_{N / N_{c}} & \cdots & \mathbf{1}_{N / N_{c}}
\end{array}\right)
$$

The Berry curvature of band $n$ is defined through the curl of connection vector from Eq. (2.3)

$$
\Omega_{n}=\nabla_{p} \times \mathcal{A}_{n n}
$$

\subsubsection{Potential}

The scalar potential $U(t, \boldsymbol{x})=[\Phi(t, \boldsymbol{x})+V(\boldsymbol{x})] E$ contains a part $\Phi$ corresponding to the ac electric field and a part $V$ corresponding to the static disorder. The scalar potential may scatter between the $N_{c}$ nodes in momentum space, this is encoded in the $N \times N$ matrix $E$. We emphasize that this does not mean that internode scattering is always important: as the nodes are centered at different momenta, the off-diagonal elements acquire a phase factor. This leads to the finite momentum Fourier transform of the potential, 
which in turn is small for smooth $U(t, \boldsymbol{x})$. For a potential $U(t, \boldsymbol{x})$ to be able to scatter between nodes $n$ and $n^{\prime}$, the Fourier component $U(t, \boldsymbol{q})$ must be sizable at $\boldsymbol{q}=\boldsymbol{b}_{n}-\boldsymbol{b}_{n^{\prime}}$.

For simplicity, we assume monochromatic ac field

$$
\Phi(t, \boldsymbol{x})=\sum_{\xi= \pm} \Phi_{\xi}(\boldsymbol{x}) e^{i \xi \omega t}, \quad \Phi_{\xi}(\boldsymbol{x})=-e \mathcal{E}_{\xi \omega} \boldsymbol{x}
$$

where $\mathcal{E}_{\xi \omega}=\mathcal{E}_{-\xi \omega}^{*}$, and discuss the generalization to arbitrary $\Phi(t, \boldsymbol{x})$ in the results section. Throughout the derivation we will treat the following two cases, see Fig. $1 \mathbf{b}$, c. In the first case, $\omega \ll E_{F}$, the frequency is small as compared to the Fermi energy $E_{F}$ i.e. all excitations are close to the Fermi surface. In the second case, $\omega>E_{F}$, the frequency is large as compared to the Fermi energy and may lead to vertical interband transitions. We consider contributions up to second order in driving fields.

The disorder potential is described by scalar impurities located at positions $\boldsymbol{R}_{i}$ that we assume to be uniformly distributed in $\mathbb{R}^{d}$ with density $n_{\text {imp }}$,

$$
V(\boldsymbol{x})=\sum_{i} \mathcal{V}\left(\boldsymbol{x}-\boldsymbol{R}_{i}\right)
$$

Our calculations are perturbative in powers of the impurity strength (see details in the next section).

\subsubsection{Wigner transform}

In addition to perturbatively weak impurity scattering, we assume an elastic scattering rate $1 / \tau \propto$ $n_{\text {imp }}|\mathcal{V}(0)|^{2} \nu(E)$, where $\nu(E)$ is the density of states), to be small as compared to the energy $E$ of the electrons. Then a semiclassical expansion is controlled.

The semiclassical phase-space quantization is conveniently expressed in terms of the Wigner transform

$$
O_{n n}(x, p)=\int_{\Delta p} O_{n n}\left(p+\frac{\Delta p}{2}, p-\frac{\Delta p}{2}\right) e^{i \Delta p_{\mu}\left(x^{\mu}-\mathcal{A}_{n}^{\mu}(p)\right)}
$$

of band-diagonal matrix elements of an arbitrary operator $\hat{O}$

$$
O_{n, n^{\prime}}\left(p ; p^{\prime}\right)=\int_{t t^{\prime}} e^{i E t-i E^{\prime} t^{\prime}}\left\langle\psi_{n, \boldsymbol{p}}|\hat{O}| \psi_{n^{\prime}, \boldsymbol{p}^{\prime}}\right\rangle=\int_{x x^{\prime}}\left\langle u_{n, \boldsymbol{p}}\left|O\left(x, x^{\prime}\right)\right| u_{n^{\prime}, \boldsymbol{p}^{\prime}}\right\rangle e^{-i x_{\mu} p^{\mu}+i x_{\mu}^{\prime} p^{\prime \mu}} e^{i \boldsymbol{b}_{n} \cdot \boldsymbol{x}-i \boldsymbol{b}_{n^{\prime}} \cdot \boldsymbol{x}} .
$$

Here we introduced $1+\mathrm{d}$ vectors $p=(E, \boldsymbol{p}), x=(t, \boldsymbol{x}), \mathcal{A}_{n}(p)=\left(0, \mathcal{A}_{n n}(\boldsymbol{p})\right)$ and used a Minkowski metric with signature $(-,+, \ldots,+)$. Note that the Wigner transform defined in Eq. (2.9) differs from the conventional Wigner transform by the explicit account of Berry connection in the exponential [104]. This ensures that $O_{n n}(x, p) \rightarrow O_{n n}(x, p)+\mathcal{O}\left(\partial_{x}^{2} \partial_{p}^{2}\right)$ is invariant under gauge transformations $\left|u_{n, p}\right\rangle \rightarrow$ $e^{i \phi_{n, p}}\left|u_{n, p}\right\rangle$ up to subleading orders in the semiclassical gradient expansion. A similar idea was first used by Altshuler [105] to account for the gauge invariance in real space $\psi(\boldsymbol{x}) \rightarrow e^{i \phi(\boldsymbol{x})} \psi(\boldsymbol{x})$ in the presence of an external electromagnetic vector potential.

As usual, the Wigner transform of convoluted operators defines the Moyal expansion (we drop the index $n n$ and phase space arguments of the functions $O(x, p), Q(x, p)$ on the right hand side for simplicity)

$$
\begin{aligned}
{[O \circ Q]_{n n}(x, p) \approx } & O(x, p) Q(x, p)+\frac{i}{2} \boldsymbol{\Omega} \cdot\left(\nabla_{x} O \times \nabla_{x} Q\right) \\
& -\frac{i}{2}\left(\partial_{t} O \partial_{E} Q-\partial_{E} O \partial_{t} Q\right)+\frac{i}{2}\left(\nabla_{x} O \nabla_{p} Q-\nabla_{p} O \nabla_{x} Q\right) .
\end{aligned}
$$

We use the symbol ' $O$ ' to denote integration/summation over repeated indices. Apart from the classical Poisson brackets we highlight the appearance of the Berry curvature in the complex, antisymmetric terms. 

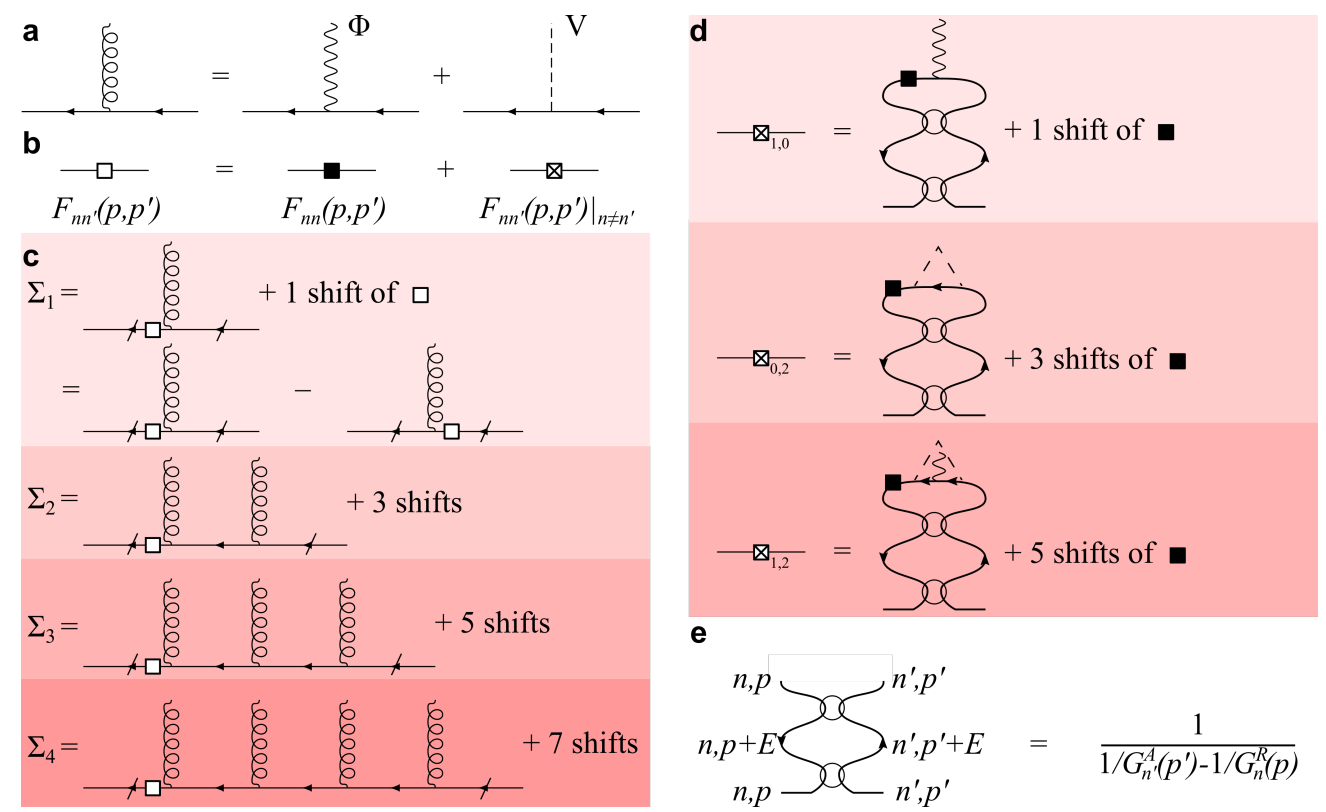

Figure 2: Illustration of the diagrammatic rules explained in the text, including the external potential (panel a) and the Keldysh operator $F$ (panel b). c Diagrammatic representation of the self energy up to fourth order in powers of $U=\Phi+V$. d Diagrammatic representation of all band off-diagonal contributions to $F_{n n^{\prime}}\left(p, p^{\prime}\right)$. e Definition of the vertex leading to the denominator $\left[G_{n^{\prime}}^{A}\left(p^{\prime}\right)\right]^{-1}-\left[G_{n}^{R}(p)\right]^{-1}$ (as obtained by taking an energy integral over $E$ and assuming that the upper loop contains one $F$ function, such that the two Green's functions inside the loop have different advanced/retarded structure). Here and in all diagrams, a slashed propagator represents an external on-shell leg.

\subsection{Dyson equation}

\subsubsection{General form and strategy}

Following the standard strategy [106], the Boltzmann equation is derived systematically from the Keldysh component of the Dyson equation (disorder average is denoted $\langle\ldots\rangle$ )

$$
-\left(\left[G^{R}\right]^{-1} \circ F-F \circ\left[G^{A}\right]^{-1}\right)=\left\langle\Sigma^{K}-\left(\Sigma^{R} \circ F-F \circ \Sigma^{A}\right)\right\rangle .
$$

Each of the operators entering Eq. (2.12) is a matrix in $N \times N$ band space, and in space-time (or equivalently, after Fourier transformation, in energy-momentum space). For example, the inverse retarded bare Green's function is $\left[G^{R}\left(p, p^{\prime}\right)\right]^{-1}=\left[E+i \eta-H_{\text {kin }}(\boldsymbol{p})\right](2 \pi)^{d+1} \delta\left(p-p^{\prime}\right)$. The operator $F$ is the unknown of the equation and, as usual, is introduced to parametrize the Keldysh component of the Green's function

$$
G^{K}=G^{R} \circ F-F \circ G^{A}
$$

The strategy to find a solution to Eq. (2.12) is a semiclassical expansion using the Wigner-transform $F(x, p)$ of which the on-shell intraband components define the distribution function.

\subsubsection{Self-energy}

The right hand side of Eq. (2.12) contains the self-energy and thereby the effective impact of the disorder potential after averaging. We also incorporate the scattering of the external electric field in the self-energy. A diagrammatic representation, before disorder average $\langle\ldots\rangle$, is given in Fig. 2, we use the notation $\left\langle\Sigma^{K}-\right.$ $\left.\left(\Sigma^{R} \circ F-F \circ \Sigma^{A}\right)\right\rangle \simeq \sum_{i=1}^{4} \Sigma_{i}$ where $i$ counts the order in external perturbation. The following Feynman rules are used.

1. An arrow on a solid line represents a Green's function $G_{n}^{R / A}$. 

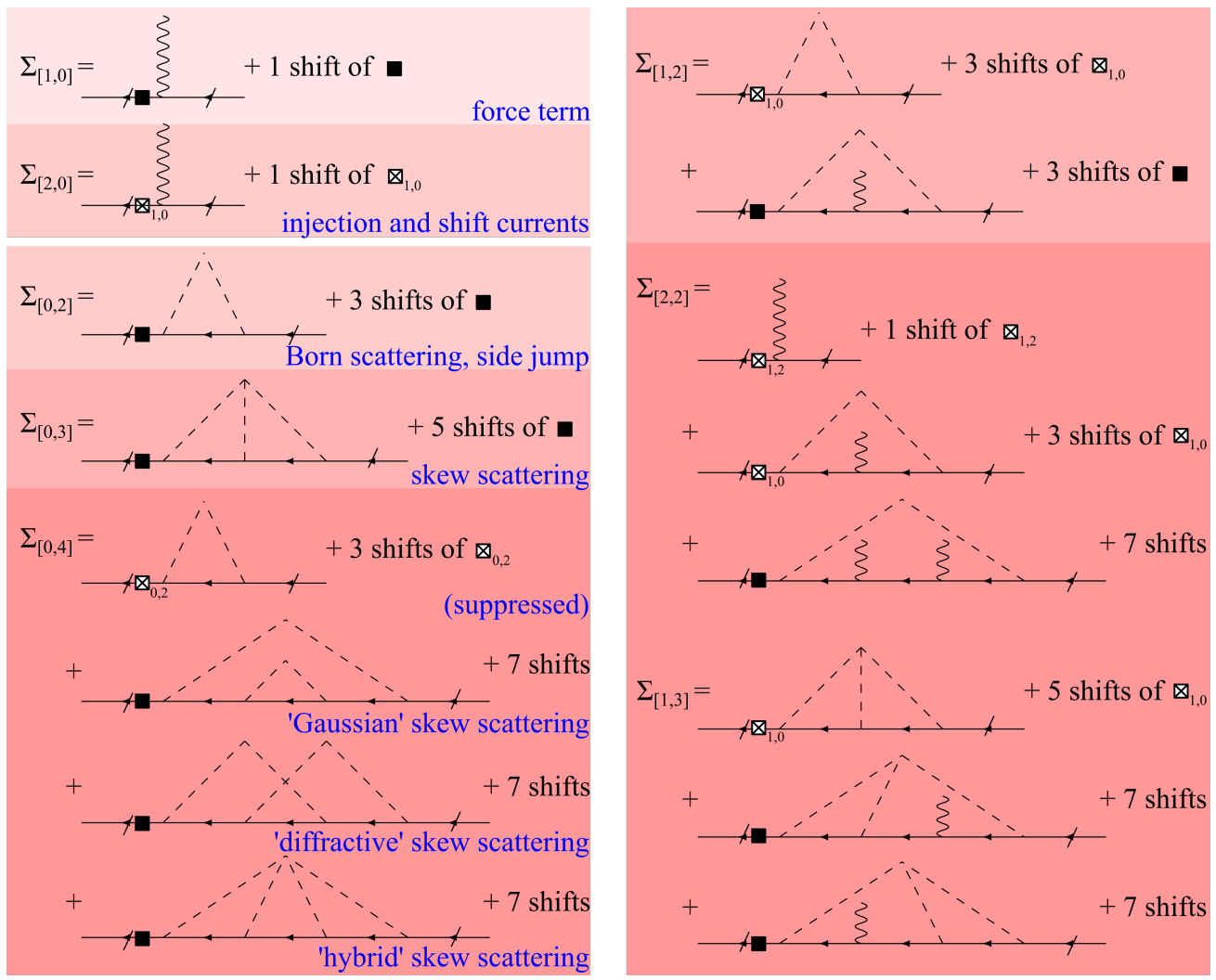

Figure 3: Effective total self energy for the intraband projected Dyson equation, Eq. (2.14), keeping only terms which are either due to photons [top left panel], only, or due to disorder, only [bottom left panel]. [Right panel]: Mixed contributions due to the self energy due to both disorder and photons.

2. An empty square on the solid line represents $F_{n n^{\prime}}\left(p, p^{\prime}\right)$, which contains band diagonal (solid box) and off-diagonal (crossed box) contributions. Per definition, all Green's functions with arrow towards (away from) the square are advanced (retarded).

3. A curly line represents $U\left(p, p^{\prime}\right)=\Phi\left(p, p^{\prime}\right)+V\left(p, p^{\prime}\right)$, where the photon is represented by a wavy line and disorder by a dashed line.

4. To $m$ th order draw all diagrams for the self-energy in the presence of $m$ curly potential lines connected by $m-1$ Green's functions. External legs are on-shell, which is represented by a dashed arrow.

5 . Generate $2 m$ diagrams by placing the empty square between all vertices and arrows (including those which are dashed). The sign of the diagram is $(-1)^{s}$ where $s=$ (number of vertices downstream) + (number of arrowheads downstream), see Fig. $2 \mathbf{b}$ for an example.

6. Take the disorder average and, as usual, keep only one particle irreducible diagrams and integrate over internal momenta.

\subsubsection{Interband Keldysh function}

While the on-shell part to intraband (i.e. diagonal) matrix elements of $F(x, p)$ corresponds to the distribution function of states in a given band, off-diagonal matrix elements $F_{n n^{\prime}}\left(n \neq n^{\prime}\right)$ are inherently quantum mechanical and appear perturbatively in powers of $V$, $\Phi$. We thus derive off-diagonal contributions to $F$ order by order, for a diagrammatic representation see Fig. 2 . In the self-energy we use the subscript $\Sigma_{[l, m]}$ to denote $l$ th order in $\mathcal{E}$ and $m$ th order in $V$. We drop the order $l=2$ which contains two small denominators representing virtual transitions. 


\subsubsection{Effective Dyson equation}

We insert this approximate solution for the off-diagonal terms into Eq. (2.12) in order to perturbatively diagonalize the equation. The result is

$$
-\frac{1}{2}\left[\left[G_{n}^{R}\right]^{-1}+\left[G_{n}^{A}\right]^{-1}, F_{n n}\right]\left(p, p^{\prime}\right)=\Sigma_{n}\left(p, p^{\prime}\right) .
$$

Here, the symbol o denotes space-time (or energy momentum) integration (without band summation) and $[\ldots, \ldots]$ is the corresponding commutator. We will henceforth omit the band index $n$ whenever possible. The effective self-energy

$$
\Sigma\left(p, p^{\prime}\right)=\sum_{\substack{l, m=1 \\ l+m \leq 4}}^{4} \Sigma_{[l, m]}\left(p, p^{\prime}\right)
$$

can be Taylor expanded, where $\Sigma_{[l, m]}\left(p, p^{\prime}\right)$ is $l$ th order in $\mathcal{E}$ and $m$ th order in $V$. We keep up to second order in electric field (as necessary for nonlinear transport) and up to fourth order in impurity scattering (which is important for skew scattering). Combined contributions are kept up to $l+m=4$. The various contributions to $\Sigma_{l, m}$ are represented in Fig: 3 , where intraband components $F_{n n}\left(p, p^{\prime}\right)$ are represented by a black box.

\subsection{Semiclassical expansion for $\omega \ll E_{F}$}

As announced above, the Boltzmann equation follows from Eq. (2.14) by a semiclassical gradient expansion. We first focus on the small frequency regime $\omega \ll E_{F}$. In this regime it is convenient to absorb the external potential into the Green's function $\mathcal{G}^{R / A}=E \pm i \eta-H_{\mathrm{kin}}(\boldsymbol{p})-\Phi(x)$. This implies that $\Sigma_{[1,0]}$ is incorporated into the left hand side of Eq. (2.14). We note that interband transitions due to photons are suppressed at small frequencies, such that $\Sigma_{[2,0]}$ is neglected along with all mixed contributions, Fig. 3, with crossed boxes. All other diagrams of Fig. 3 (right panel) are accounted for by replacing Green's functions $G^{R / A}$ by $\mathcal{G}^{R / A}$ in Fig. 3 (left panels). We define the distribution function by enforcing the on-shell constraint on diagonal components $F_{n n}(x, p)$

$$
f_{n}(t, \boldsymbol{x}, \boldsymbol{p})=\int_{E} \delta\left(E-E_{n}(\boldsymbol{p})-\Phi(x)\right) F_{n n}(x, p) .
$$

\subsubsection{Liouville term}

Using the Moyal expansion derived in Eq. (2.11) we readily obtain the Liouvillian term describing the reactive response of the system

$$
\begin{aligned}
-\frac{1}{2}\left[\left[\mathcal{G}_{n}^{R}\right]^{-1}+\left[\mathcal{G}_{n}^{A}\right]^{-1}, F_{n n}\right](x, p) & \approx-i\left(\left[\partial_{t}+\partial_{t} \Phi \partial_{E}\right] F_{n n}(x, p)\right. \\
& \left.+\left[\boldsymbol{v}_{n}-\boldsymbol{\Omega}_{n} \times \nabla_{x} \Phi\right] \cdot \nabla_{\boldsymbol{x}} F_{n n}(x, p)-\nabla_{x} \Phi \cdot \nabla_{p} F_{n n}(x, p)\right),
\end{aligned}
$$

where the band velocity is $\boldsymbol{v}_{n}=\nabla_{p} E_{n}(\boldsymbol{p})$. Clearly it follows then

$$
-\frac{i}{2} \int_{E} \delta\left(E-E_{n}(\boldsymbol{p})-\Phi(x)\right)\left[\left[\mathcal{G}_{n}^{R}\right]^{-1}+\left[\mathcal{G}_{n}^{A}\right]^{-1}, F_{n n}\right](x, p) \approx\left(\partial_{t}+\dot{\boldsymbol{p}} \cdot \nabla_{\boldsymbol{p}}+\dot{\boldsymbol{x}} \cdot \nabla_{\boldsymbol{x}}\right) f_{n}(t, \boldsymbol{x}, \boldsymbol{p}),
$$

which reproduces the usual appearance of the Liouville term in the Boltzmann equation, where $\dot{\boldsymbol{x}}=\boldsymbol{v}_{n}+$ $\boldsymbol{\Omega}_{n} \times \dot{\boldsymbol{p}}$, and $\dot{\boldsymbol{p}}=-\nabla_{x} \Phi$. 


\subsubsection{Born scattering and side jump}

The diagram for these processes is depicted in Fig. 3 (top left panel) and its analytical expression in energy-momentum space is

$$
\begin{aligned}
\Sigma_{[0,2]}\left(p_{+}, p_{-}\right) & =n_{\mathrm{imp}} \oint_{n^{\prime} \boldsymbol{p}^{\prime}} F_{n n}\left(p_{+}, p_{-}\right)\left|\mathcal{V}\left(\left(\boldsymbol{p}-\boldsymbol{b}_{n}\right)-\left(\boldsymbol{p}^{\prime}-\boldsymbol{b}_{n^{\prime}}\right)\right)\right|^{2} \\
& \times\left[\left|\left\langle u_{n, \boldsymbol{p}_{-}}|E| u_{n^{\prime}, \boldsymbol{p}_{-}^{\prime}}\right\rangle\right|^{2} \mathcal{G}_{n^{\prime}}^{A}\left(E_{-}, \boldsymbol{p}_{-}^{\prime}\right)-\left|\left\langle u_{n, \boldsymbol{p}_{+}}|E| u_{n^{\prime}, \boldsymbol{p}_{+}^{\prime}}\right\rangle\right|^{2} \mathcal{G}_{n^{\prime}}^{R}\left(E_{+}, \boldsymbol{p}_{+}^{\prime}\right)\right] \\
& -n_{\mathrm{imp}} \oint_{n^{\prime} \boldsymbol{p}^{\prime}} F_{n^{\prime} n^{\prime}}\left(E_{+}, \boldsymbol{p}_{+}^{\prime} ; E_{-}, \boldsymbol{p}_{-}^{\prime}\right) \mid \mathcal{V}\left(\left(\boldsymbol{p}-\boldsymbol{b}_{n}\right)-\left(\boldsymbol{p}^{\prime}-\boldsymbol{b}_{n^{\prime}}\right)\right) \\
& \times\left.\left\langle u_{n, \boldsymbol{p}_{+}}|E| u_{n^{\prime}, \boldsymbol{p}_{+}^{\prime}}\right\rangle\left\langle u_{n^{\prime}, \boldsymbol{p}_{-}^{\prime}}|E| u_{n, \boldsymbol{p}_{+}}\right\rangle\right|^{2}\left[\mathcal{G}_{n^{\prime}}^{A}\left(E_{-}, \boldsymbol{p}_{-}^{\prime}\right)-\mathcal{G}_{n^{\prime}}^{R}\left(E_{+}, \boldsymbol{p}_{+}^{\prime}\right)\right] .
\end{aligned}
$$

Here we introduced a joint integration symbol $\oint$ that implies summation over the discrete index $\sum_{n^{\prime}}$ and integration over the continuous variable $\int_{p^{\prime}}$. We expand the above expression in small $\Delta p$ and use the Wigner transform, to obtain

$$
\begin{aligned}
\Sigma_{[0,2]}(x, p) & \approx i n_{\text {imp }} \oint_{n^{\prime} \boldsymbol{p}^{\prime}}(2 \pi)^{2} \delta\left(E-E_{n^{\prime}}\left(\boldsymbol{p}^{\prime}\right)\right) \delta\left(E-E^{\prime}\right)\left|\mathcal{V}\left(\left(\boldsymbol{p}-\boldsymbol{b}_{n}\right)-\left(\boldsymbol{p}^{\prime}-\boldsymbol{b}_{n^{\prime}}\right)\right)\right|^{2}\left|\left\langle u_{n, \boldsymbol{p}}|E| u_{n^{\prime}, \boldsymbol{p}^{\prime}}\right\rangle\right|^{2} \\
& \times\left[F_{n n}(x, p)-\left[1+\delta \boldsymbol{r}_{n^{\prime} n}\left(\boldsymbol{p}^{\prime}, \boldsymbol{p}\right) \nabla_{\boldsymbol{x}}\right] F_{n^{\prime} n^{\prime}}\left(x, p^{\prime}\right)\right] .
\end{aligned}
$$

Here, we dropped principle value integrals (suppressed virtual processes) and introduced the displacement at a side-jump $[28,29]$

$$
\delta \boldsymbol{r}_{n^{\prime} n}\left(\boldsymbol{p}^{\prime}, \boldsymbol{p}\right)=\mathcal{A}_{n^{\prime}}\left(\boldsymbol{p}^{\prime}\right)-\mathcal{A}_{n}(\boldsymbol{p})-\left(\nabla_{\boldsymbol{p}}+\nabla_{\boldsymbol{p}^{\prime}}\right) \arg \left(\left\langle u_{n,,^{\prime} \boldsymbol{p}^{\prime}}|E| u_{n, \boldsymbol{p}}\right\rangle\right) .
$$

After projecting onto the mass shell, we obtain the collision integral ("Stossintegral" in German) of the right-hand-side in the Boltzmann equation

$$
\begin{aligned}
\operatorname{St}_{\mathrm{Born}}\{f\}+\mathrm{St}_{\mathrm{sj}}\{f\} & =-2 \pi n_{\mathrm{imp}} \oint_{n^{\prime} \boldsymbol{p}^{\prime}} \delta\left(E_{n}(\boldsymbol{p})+\Phi(t, \boldsymbol{x})-E_{n^{\prime}}\left(\boldsymbol{p}^{\prime}\right)-\Phi\left(t, \boldsymbol{x}+\delta \boldsymbol{r}_{n^{\prime} n}\left(\boldsymbol{p}^{\prime}, \boldsymbol{p}\right)\right)\right) \\
& \left|\mathcal{V}\left(\left(\boldsymbol{p}-\boldsymbol{b}_{n}\right)-\left(\boldsymbol{p}^{\prime}-\boldsymbol{b}_{n^{\prime}}\right)\right)\right|^{2}\left|\left\langle u_{n, \boldsymbol{p}}|E| u_{n^{\prime}, \boldsymbol{p}^{\prime}}\right\rangle\right|^{2}\left[f_{n}(t, \boldsymbol{x}, \boldsymbol{p})-f_{n^{\prime}}\left(t, \boldsymbol{x}+\delta \boldsymbol{r}_{n^{\prime} n}\left(\boldsymbol{p}^{\prime}, \boldsymbol{p}\right), \boldsymbol{p}^{\prime}\right)\right] .
\end{aligned}
$$

We highlight that this expression, which contains both Born scattering $\operatorname{St}_{\text {Born }}\{f\}$ (Eq. $(2.22)$ at $\delta \boldsymbol{r}=0$ ) and the side-jump effect $\mathrm{St}_{\mathrm{sj}}\{f\}$ (Eq. (2.22) to first order in $\delta \boldsymbol{r}=0$ ), is to be understood up to first order in the gradient expansion. We remind that in common notations, the latter collision term $\operatorname{St}_{\text {sj }}\{f\}$ is often referred to as anomalous distribution.

\subsubsection{Skew scattering}

It will be useful to use a multi-index notation $l=(n, \boldsymbol{p})$ as well as energy notation $E_{l}=E_{n}(\boldsymbol{p})$, and introduce matrix elements $V_{l l^{\prime}}=\left\langle u_{n, \boldsymbol{p}}|E| u_{n^{\prime}, \boldsymbol{p}^{\prime}}\right\rangle V\left(\left(\boldsymbol{p}-\boldsymbol{b}_{n}\right)-\left(\boldsymbol{p}^{\prime}-\boldsymbol{b}_{n^{\prime}}\right)\right)$. In this notation, we find prior to disorder average

$$
\begin{aligned}
\Sigma_{[0,3]}(x, p) & =\oint_{l_{1}, l_{2}} F_{n n}(x, p) V_{l l_{1}} V_{l_{1} l_{2}} V_{l_{2} l}\left[\mathcal{G}_{l_{1}}^{A} \mathcal{G}_{l_{2}}^{A}-\mathcal{G}_{l_{1}}^{R} \mathcal{G}_{l_{2}}^{R}\right] \\
& +F_{n_{1} n_{1}}\left(x, p_{1}\right)\left[\mathcal{G}_{l_{1}}^{R}-\mathcal{G}_{l_{1}}^{A}\right]\left[V_{l l_{1}} V_{l_{1} l_{2}} V_{l_{2} l} \mathcal{G}_{l_{2}}^{A}+V_{l l_{2}} V_{l_{2} l_{1}} V_{l_{1} l} \mathcal{G}_{l_{2}}^{R}\right] .
\end{aligned}
$$

We emphasize that all Green's function have the same energy argument since impurity scattering is elastic. We only need contributions which are odd under $l \leftrightarrow l_{1}$ and obtain, after on-shell projection, the skew scattering collision term in the form

$$
\operatorname{St}_{\mathrm{sk}}\{f\}=\oint_{l^{\prime}} W_{l l^{\prime}}^{\mathrm{sk}} f_{l^{\prime}}
$$


where the corresponding transition probability is

$$
W_{l l^{\prime}}^{\mathrm{sk}}=-\oint_{l_{2}}(2 \pi)^{2} \delta\left(E_{l}-E_{l^{\prime}}\right) \delta\left(E_{l}-E_{l_{2}}\right) \operatorname{Im}\left[V_{l l^{\prime}} V_{l^{\prime} l_{2}} V_{l_{2} l}\right] .
$$

With additional simplifying assumptions further analytical progress can be made. For instance, one tractable example corresponds to a disorder model created by a centrosymmetric impurity potential, $\mathcal{V}(\boldsymbol{x})=$ $\mathcal{V}(-\boldsymbol{x})$, that is assumed to vary slowly on the scale of the lattice constant. For this model, the electron transition matrix element separates into the product of a Fourier transform of the impurity potential and a Bloch wave function overlap of states within the same node, $\propto \mathcal{V}\left(\boldsymbol{p}_{1}-\boldsymbol{p}_{2}\right)\left\langle u_{l_{1}} \mid u_{l_{2}}\right\rangle \simeq V_{0}\left\langle u_{l_{1}} \mid u_{l_{2}}\right\rangle$, where $V_{0}$ denotes the strength of intranode scattering. Since for a centrosymmetric impurity $\operatorname{Im}\left[V_{p}\right]=0$, the antisymmetric part of the scattering probability defined by Eq. (2.24) becomes after disorder average

$$
W_{l l^{\prime}}^{\mathrm{sk}}=-n_{\mathrm{imp}} V_{0}^{3} \oint_{l^{\prime \prime}}(2 \pi)^{2} \delta\left(E_{l}-E_{l^{\prime}}\right) \delta\left(E_{l}-E_{l^{\prime \prime}}\right) Z_{l l^{\prime} l^{\prime \prime}}
$$

where

$$
Z_{l_{1} l_{2} l_{3}}=\operatorname{Im}\left[\left\langle u_{l_{1}} \mid u_{l_{2}}\right\rangle\left\langle u_{l_{2}} \mid u_{l_{3}}\right\rangle\left\langle u_{l_{3}} \mid u_{l_{1}}\right\rangle\right]
$$

This contribution appears from third order scattering of a single impurity, see Fig. 3.

\subsubsection{Gaussian, diffractive, and hybrid skew scattering}

The first line of $\Sigma_{[0,4]}$ in Fig. 3 is small in powers of the semiclassical parameter of Fermi energy being much larger than the elastic scattering rate. This follows, because both the crossed box and the Green's function account for virtual elastic (i.e. horizontal) interband transitions. Prior to disorder average, we thus find

$$
\begin{aligned}
\Sigma_{[0,4]}(x, p) & =\oint_{l^{\prime}, l_{1}, l_{2}} F_{n n}(x, p) V_{l l^{\prime}} V_{l^{\prime} l_{1}} V_{l_{1} l_{2}} V_{l_{2} l}\left[\mathcal{G}_{l^{\prime}}^{A} \mathcal{G}_{l_{1}}^{A} \mathcal{G}_{l_{2}}^{A}-\mathcal{G}_{l^{\prime}}^{R} \mathcal{G}_{l_{1}}^{R} \mathcal{G}_{l_{2}}^{R}\right]+F_{n^{\prime} n^{\prime}}\left(x, p^{\prime}\right)\left[\mathcal{G}_{l^{\prime}}^{R}-\mathcal{G}_{l^{\prime}}^{A}\right] \\
& \times\left\{V_{l l^{\prime}} V_{l^{\prime} l_{1}} V_{l_{1} l_{2}} V_{l_{2} l} \mathcal{G}_{l_{1}}^{A} \mathcal{G}_{l_{2}}^{A}+V_{l l_{1}} V_{l_{1} l_{2}} V_{l_{2} l^{\prime}} V_{l^{\prime} l} \mathcal{G}_{l_{1}}^{R} \mathcal{G}_{l_{2}}^{R}+V_{l l_{1}} V_{l_{1} l^{\prime}} V_{l^{\prime} l_{2}} V_{l_{2} l} \mathcal{G}_{l_{1}}^{R} \mathcal{G}_{l_{2}}^{A}\right\} .
\end{aligned}
$$

Again, we only keep the contribution which is odd under $l \leftrightarrow l^{\prime}$ and project on-shell to obtain

$$
\mathrm{St}_{\mathrm{sk}}^{[4]}[f]=\oint_{l^{\prime}} \widetilde{W}_{l l^{\prime}}^{\mathrm{sk}} f_{l^{\prime}}
$$

where

$$
\begin{aligned}
\widetilde{W}_{l l^{\prime}}^{\mathrm{sk}} & =-\oint_{l_{1}, l_{2}}(2 \pi)^{2} \delta\left(E_{l}-E_{l_{1}}\right) \delta\left(E_{l}-E_{l^{\prime}}\right)\left[\mathcal{G}_{l_{2}}^{R}+\mathcal{G}_{l_{2}}^{A}\right] / 2 \\
& \times\left\{\operatorname{Im}\left[V_{l l^{\prime}} V_{l^{\prime} l_{1}} V_{l_{1} l_{2}} V_{l_{2} l}+(1 \leftrightarrow 2)\right]+2 \operatorname{Im}\left[V_{l l_{2}} V_{l_{2} l^{\prime}} V_{l^{\prime} l_{1}} V_{l_{1} l}\right]\right\} .
\end{aligned}
$$

Note that contrary contrary to all previous contributions, here one off-shell contribution $\left[\mathcal{G}_{l_{2}}^{R}+\mathcal{G}_{l_{2}}^{A}\right]$ is explicit. Disorder average implies the impurities leading to three different kinds of diagrams, see Fig. 3: scattering from two different impurities allows for a rainbow diagram (Gaussian skew scattering) [29] and a crossed diagram (diffractive skew scattering) [61, 62], while scattering from a single impurity to fourth order leads to hybrid skew scattering [59].

We illustrate these three contributions, $\widetilde{W}_{l l^{\prime}}^{\text {sk }}=W_{l l^{\prime}}^{\text {Gauss }}+W_{l l^{\prime}}^{\text {diff }}+W_{l l^{\prime}}^{\text {hybrid }}$ again in the limit of a smooth, centrosymmetric impurity potential of intranode scattering strength $V_{0}$. After impurity average, the expres- 
sions simplify to

$$
\begin{aligned}
W_{l l^{\prime}}^{\text {Gauss }} & =-\left(n_{\mathrm{imp}} V_{0}\right)^{2} \oint_{l_{1}, l_{2}} 2 \pi^{2} \delta\left(E_{l}-E_{l_{1}}\right) \delta\left(E_{l}-E_{l^{\prime}}\right)\left[\mathcal{G}_{l_{2}}^{R}+\mathcal{G}_{l_{2}}^{A}\right] \\
& \times\left\{\left(Z_{l l^{\prime} l_{1} l_{2}} \delta_{p_{2}, \boldsymbol{p}^{\prime}}+Z_{l l^{\prime} l_{2} l_{1}} \delta_{\boldsymbol{p}_{1}, \boldsymbol{p}^{\prime}}\right)+2 Z_{l l_{2} l^{\prime} l_{1}} \delta_{\boldsymbol{p}_{1}, \boldsymbol{p}_{2}}\right\}, \\
W_{l l^{\prime}}^{\mathrm{diff}} & =-\left(n_{\mathrm{imp}} V_{0}\right)^{2} \oint_{l_{1}, l_{2}} 2 \pi^{2} \delta\left(E_{l}-E_{l_{1}}\right) \delta\left(E_{l}-E_{l^{\prime}}\right)\left[\mathcal{G}_{l_{2}}^{R}+\mathcal{G}_{l_{2}}^{A}\right] \\
& \left.\times\left\{\left(Z_{l l^{\prime} l_{1} l_{2}} \delta_{\boldsymbol{p}_{2}-\boldsymbol{p}_{1}, \boldsymbol{p}-\boldsymbol{p}^{\prime}}+1 \leftrightarrow 2\right)\right)+2 Z_{l l_{2} l^{\prime} l_{1}} \delta_{p_{1}+\boldsymbol{p}_{2}, \boldsymbol{p}+\boldsymbol{p}^{\prime}}\right\}, \\
W_{l l^{\prime}}^{\text {hybrid }} & =-n_{\mathrm{imp}}\left(V_{0}\right)^{4} \oint_{l_{1}, l_{2}} 2 \pi^{2} \delta\left(E_{l}-E_{l_{1}}\right) \delta\left(E_{l}-E_{l^{\prime}}\right)\left[\mathcal{G}_{l_{2}}^{R}+\mathcal{G}_{l_{2}}^{A}\right]\left\{\left(Z_{l l^{\prime} l_{1} l_{2}}+1 \leftrightarrow 2\right)+2 Z_{l l_{2} l^{\prime} l_{1}}\right\},
\end{aligned}
$$

where we introduce the four index analog to Eq. (2.24d)

$$
Z_{l_{1}, l_{2}, l_{3}, l_{4}}=\operatorname{Im}\left[\left\langle u_{l_{1}} \mid u_{l_{2}}\right\rangle\left\langle u_{l_{2}} \mid u_{l_{3}}\right\rangle\left\langle u_{l_{3}} \mid u_{l_{4}}\right\rangle\left\langle u_{l_{4}} \mid u_{l_{1}}\right\rangle\right] \text {. }
$$

We conclude the section on skew scattering from the fourth order potential with a technical remark relating to the diagrammatic calculation of the anomalous Hall conductivity bubble. In the equation for $W_{l l^{\prime}}^{\text {diff }}$, the first two terms in the curly brackets represent so called $\Psi$ diagrams, while the last term is the $X$ diagram (in the notation of $[61,62,64,65]$ ).

\subsubsection{Summary small frequency result}

This concludes the derivation of the Boltzmann equation in Berry curved matter in the low-frequency $\left(\omega \ll E_{F}\right.$ limit $)$ :

$$
\left[\partial_{t}+\dot{\boldsymbol{x}} \cdot \nabla_{\boldsymbol{x}}+\dot{\boldsymbol{p}} \cdot \nabla_{\boldsymbol{p}}\right] f(t, \boldsymbol{x}, \boldsymbol{p})=\operatorname{St}\{f\},
$$

where $\dot{\boldsymbol{x}}=\boldsymbol{v}_{n}+\boldsymbol{\Omega}_{n} \times \dot{\boldsymbol{p}}, \dot{\boldsymbol{p}}=-\nabla_{\boldsymbol{x}} \Phi$ and the collision integral St $[f]=\operatorname{St}_{\mathrm{Born}}\{f\}+\operatorname{St}_{\mathrm{sj}}\{f\}+\operatorname{St}_{\mathrm{sk}}\{f\}+\operatorname{St}_{\mathrm{sk}}^{[4]}\{f\}$ is given by Eqs. (2.22), (2.24), (2.26). We explicitly kept non-linear orders of the external potential in the collision integral (which eventually vanish), but restricted ourselves to terms of zeroth and first order in gradients $\left(\hbar \partial_{x} \partial_{p}\right)$, and dropped the combination of skew and side-jump effects.

\subsection{Semiclassical expansion for $\omega \gtrsim E_{F}$}

In this section we derive the effective kinetic theory for an external field with fast driving frequency. We concentrate on the rectified current stemming from slowly fluctuating $F_{n n}(t, \boldsymbol{x} ; E, \boldsymbol{p})$ for which a semiclassical gradient expansion is justified, while we omit quickly oscillating first and second harmonics of $F_{n n}(t, \boldsymbol{x} ; E, \boldsymbol{p})$. The physical reason behind concentrating on the rectified current within the present Boltzmann technique is that the latter is mainly designed to incorporate relaxation effects. On the other hand, relaxation is negligible for the quickly oscillating first and second harmonic.

Technically, we time average Eq. $(2.14)\langle\ldots\rangle_{\text {time }}=\int_{0}^{2 \pi / \omega}[d t \ldots] \omega / 2 \pi$, whereby the terms $\Sigma_{1,0}, \Sigma_{1,2}, \Sigma_{1,3}$ vanish. Since the external potential is quickly oscillating, we use a slightly different definition of the distribution function than in the slow frequency limit (cf. Eq. (2.16))

$$
f_{n}(t, \boldsymbol{x}, \boldsymbol{p})=\int_{E} \delta\left(E-E_{n}(\boldsymbol{p})\right) F_{n n}(x, p) .
$$

\subsubsection{Liouville term and disorder scattering}

The Liouville term and disorder induced collision integrals for the rectified distribution function are essentially the same as in the slow-frequency limit, Eqs. (2.18), (2.22), (2.24), (2.26), and follow from analogous derivations. The only major difference is the cancellation of linear terms in the external potential leading to absent force term, $\dot{\boldsymbol{p}}=0$, and side jump contributions. 


\subsubsection{Injection and shift currents}

The vertical interband scattering off two photons leads to a self-energy contribution

$$
\begin{aligned}
\Sigma_{[2,0]}(x, p) \simeq i \sum_{n^{\prime}} \sum_{i, j=1}^{d} \sum_{\xi= \pm} & 2 \pi \delta\left(\xi \omega+E_{n^{\prime}}(\boldsymbol{p})-E_{n}(\boldsymbol{p})\right) e^{2} \mathcal{E}_{\xi}^{i} \mathcal{E}_{-\xi}^{j} \mathcal{A}_{n n^{\prime}}^{i}(\boldsymbol{p}) \mathcal{A}_{n^{\prime} n}^{j}(\boldsymbol{p}) \\
\times & \left.\times F_{n n}(x, p)-\left[1+\boldsymbol{R}_{n^{\prime} n}(\boldsymbol{p}) \cdot \nabla_{\boldsymbol{x}}\right] F_{n^{\prime} n^{\prime}}(x, p-\xi \omega)\right\} .
\end{aligned}
$$

Here we used a suggestive notation $p-\xi \omega=(E-\xi \omega, \boldsymbol{p})$. On a technical level, the derivation is very similar to the derivation of Eq. (2.20) presented above. Again, the scattering process is accompanied by a coordinate shift, which for photons reads [28]

$$
\boldsymbol{R}_{n^{\prime} n}(\boldsymbol{p})=\mathcal{A}_{n^{\prime}}(\boldsymbol{p})-\mathcal{A}_{n}(\boldsymbol{p})-\nabla_{p} \arg \left(\mathcal{A}_{n^{\prime} n}(\boldsymbol{p})\right) .
$$

The projection of this equation on the mass shell generates a collision "integral" (a source term for photocarrier injection)

$$
\begin{array}{r}
\operatorname{St}_{\mathrm{PGE}}\{f\}=-\sum_{n^{\prime}} \sum_{i, j=1}^{d} \sum_{\xi= \pm} 2 \pi \delta\left(\xi \omega+E_{n^{\prime}}(\boldsymbol{p})-E_{n}(\boldsymbol{p})\right) e^{2} \mathcal{E}_{\xi}^{i} \mathcal{E}_{-\xi}^{j} \mathcal{A}_{n n^{\prime}}^{i}(\boldsymbol{p}) \mathcal{A}_{n^{\prime} n}^{j}(\boldsymbol{p}) \\
\times\left\{f_{n}(t, \boldsymbol{x}, \boldsymbol{p})-\left[1+\boldsymbol{R}_{n^{\prime} n}(\boldsymbol{p}) \cdot \nabla_{\boldsymbol{x}}\right] f_{n^{\prime}}(t, \boldsymbol{x}, \boldsymbol{p})\right\} .
\end{array}
$$

\subsubsection{Mixed disorder and photon scattering}

The interplay of disorder and optical excitation is encoded in $\Sigma_{[2,2]}(x, p)$. To leading order, there are two impurity induced contributions for optical transitions.

First, a particle in the filled valence band first scatters off an impurity to a virtual off-shell state, and then performs an optically allowed transition. Second, the reverse happens, i.e. an optical transition to an off-shell state in the conduction band, and an impurity assisted scattering to an on-shell final state in the conduction band. The contribution $\Sigma_{[2,2]}(x, p)$ describes these transitions and we leave a careful study to a separate publication.

We remark that $\Sigma_{[1,3]}(x, p)$ vanishes upon time average (as mentioned, this is the only relaxation prone channel in the large frequency limit). Therefore interband contributions linear in external field, as discussed in Ref. [81], are disregarded.

\subsubsection{Summary of large frequency result}

In total, the kinetic equation at large frequencies takes the form

$$
\left[\partial_{t}+\dot{\boldsymbol{x}} \cdot \nabla_{x}\right] f(t, \boldsymbol{x}, \boldsymbol{p})=\operatorname{St}\{f\},
$$

where $\dot{\boldsymbol{x}}=\boldsymbol{v}_{n}$ and $\operatorname{St}\{f\}$ has contributions from the injection of photocarriers, Eq. (2.31), relaxation and skew scattering effects at impurities, Eqs. (2.22), (2.24), (2.26), as well as mixed contributions to be discussed elsewhere.

Note that the kinetic Eq. (2.32) contains a carrier injection far away from the Fermi surface - here the energy of on-shell particles does not necessarily coincide with the Fermi level and interactions induce inelastic energy relaxation effects necessary for photocarrier decay.

\section{Electrical current response}

In this section we present derivation of the electrical current response and elucidate the origin of the side-jump accumulation velocity.

In the field integral representation of the Keldysh technique [106], the electrical current is defined by a functional derivative of the partition function, $j_{i}(x)=\left.\left[\partial Z / \partial A_{\mathrm{q}}^{i}(x)\right]\right|_{\boldsymbol{A}_{\mathrm{q}}=0}$, where the index-q indicates 

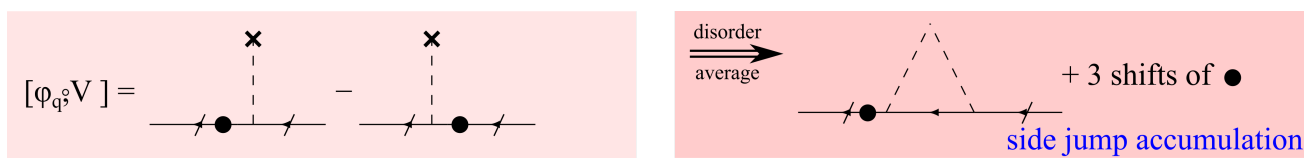

Figure 4: Renormalization of the current vertex by the impurity potential. In the disorder contribution to the commutator Eq. (3.2), the U(1) phase $\phi_{\mathrm{q}}$ is represented by a disk.

the quantum component of the vector potential. The coupling of the latter to the fields is imposed by electromagnetic U(1) gauge invariance. Therefore, the current operator associated to Bloch-electrons of band $n$ can be obtained from the gauge transformation of

$$
\hat{G}_{n n}^{-1}=\left(\begin{array}{cc}
{\left[\hat{G}^{R}\right]^{-1}} & {\left[\hat{G}^{-1}\right]^{K}} \\
0 & {\left[\hat{G}^{A}\right]^{-1}}
\end{array}\right)_{n n} \rightarrow e^{-i \phi_{\mathrm{q}}(x) \hat{\gamma}_{\mathrm{q}}} \hat{G}_{n n}^{-1} e^{i \phi_{\mathrm{q}}(x) \hat{\gamma}_{\mathrm{q}}}
$$

Here, following standard convention, $\hat{\gamma}_{\mathrm{q}}$ is the first Pauli matrix in Keldysh space. To leading order

$$
\delta \hat{G}_{n n}^{-1}=i\left[\phi_{\mathrm{q}}^{\circ}, \hat{H}_{n n}\right] \hat{\gamma}_{\mathrm{q}}
$$

where $\hat{H}_{n n}$ is the full Hamiltonian prior to disorder average and projected on band $n$. We omitted terms which are stemming from the regulation of Keldysh theory (e.g. $\left[\hat{G}^{R}\right]^{-1}-\left[\hat{G}^{A}\right]^{-1} \sim i \eta \rightarrow 0$ ).

For the clean part of $\hat{H}_{n n}$ we use the Wigner transform Eq. $(2.9)$ to obtain $\left.\delta \hat{G}_{n n}^{-1}(x, p)\right|_{V=0} \approx-\dot{\boldsymbol{x}} \cdot \nabla_{\boldsymbol{x}} \phi_{q} \hat{\gamma}_{\mathrm{q}}$, where $\dot{\boldsymbol{x}}=\boldsymbol{v}+\boldsymbol{\Omega} \times \dot{\boldsymbol{p}}$. The contribution from the disorder potential contains a renormalization of the vertex depicted diagrammatically in Fig. 4 . Here, the matrix $\hat{\gamma}_{\mathrm{q}}$ imposes that Green's functions which are coming into (going out of) the vertex are advanced (retarded). The similarity of this diagrams with $\Sigma_{[0,2]}$ in Fig. 3 , where the square representing $F_{n n}(x, p)$ is replaced by the disk representing $\phi_{q}(x)$, implies that the vertex renormalization is given in complete analogy to Eq. (2.20) by

$$
\begin{aligned}
\left\langle\delta \hat{G}_{n n}^{-1}(x, p)\right\rangle & \approx-n_{\mathrm{imp}} \oint_{n^{\prime} \boldsymbol{p}^{\prime}}(2 \pi) \delta\left(E-E_{n^{\prime}}\left(\boldsymbol{p}^{\prime}\right)\right) \delta \boldsymbol{r}_{n^{\prime} n}\left(\boldsymbol{p}^{\prime}, \boldsymbol{p}\right) \nabla_{\boldsymbol{x}} \phi_{\mathrm{q}} \\
& \times\left|\mathcal{V}\left(\left(\boldsymbol{p}-\boldsymbol{b}_{n}\right)-\left(\boldsymbol{p}^{\prime}-\boldsymbol{b}_{n^{\prime}}\right)\right)\right|^{2}\left|\left\langle u_{n, \boldsymbol{p}}|E| u_{n^{\prime}, \boldsymbol{p}^{\prime}}\right\rangle\right|^{2} \hat{\gamma}^{q} .
\end{aligned}
$$

The average current is thus

$$
\boldsymbol{j}=e \int_{\boldsymbol{p}}\left[\dot{\boldsymbol{x}}+\boldsymbol{v}^{\mathrm{sj}}\right] f_{n}(t, \boldsymbol{x}, \boldsymbol{p}) .
$$

Here, we introduced the side jump accumulation velocity

$$
\boldsymbol{v}^{\mathrm{sj}}=n_{\mathrm{imp}} \oint_{n^{\prime} \boldsymbol{p}^{\prime}}(2 \pi) \delta\left(E-E_{n^{\prime}}\left(\boldsymbol{p}^{\prime}\right)\right) \delta \boldsymbol{r}_{n^{\prime} n}\left(\boldsymbol{p}^{\prime}, \boldsymbol{p}\right)\left|\mathcal{V}\left(\left(\boldsymbol{p}-\boldsymbol{b}_{n}\right)-\left(\boldsymbol{p}^{\prime}-\boldsymbol{b}_{n^{\prime}}\right)\right)\right|^{2}\left|\left\langle u_{n, \boldsymbol{p}}|E| u_{n^{\prime}, \boldsymbol{p}^{\prime}}\right\rangle\right|^{2} .
$$

In the case of large frequencies, we omit the photon field in $\dot{x}_{i}$, as it is only important for intraband physics.

\section{Anomalous Hall transport of multifold fermions}

In this section we present an application of our theory for the anomalous transport in the model of multifold fermions, as defined in Eq. (1.1). We remind that for a spin- $S$ the corresponding operators are square matrices of dimension $2 S+1$. They can be conveniently represented in the Zeeman basis with states labeling $|S, m\rangle$. We choose to work in this basis. 


\subsection{Eigenstates and Berry curvature}

As the starting point in obtaining the Berry curvature for this model it is convenient to use spherical coordinates for the $\boldsymbol{d}$-vector

$$
\boldsymbol{d}=d(\sin (\theta) \cos (\phi), \sin (\theta) \sin (\phi), \cos (\theta))^{T} .
$$

The eigenstates of Eq. (1.1) then readily follow from rotating the Hamiltonian onto the $\hat{z}$ axis, i.e.

$$
\left|u_{m, \boldsymbol{p}}\right\rangle=e^{-i \phi S_{z}} e^{-i \theta S_{y}} \hat{e}_{m}
$$

where $\hat{e}_{m}$ is the unit vector pointing in $m$ direction, with the conventional labeling of $m=-S,-S+$ $1, \ldots, S-1, S$. The energy of these states is $E_{m}(\boldsymbol{p})=d_{0}(\boldsymbol{p})+m d(\boldsymbol{p})$. From this definition, the Berry curvature can be now computed from the standard formula

$$
\Omega_{a}=i \varepsilon_{a b c}\left\langle\partial_{b} u_{m, p} \mid \partial_{c} u_{m, p}\right\rangle=m \varepsilon_{a b c} \partial_{b} \phi \partial_{c} \theta \sin (\theta)=-\frac{m}{2} \varepsilon_{a b c} \hat{d} \cdot\left(\partial_{b} \hat{d} \times \partial_{c} \hat{d}\right) .
$$

where the unit vector is $\hat{d}=\boldsymbol{d} / d$.

\subsection{Projectors on eigenstates}

As we shall shortly see, the projectors of eigenstates

$$
\hat{P}_{m, \boldsymbol{p}} \equiv\left|u_{m, \boldsymbol{p}}\right\rangle\left\langle u_{m, \boldsymbol{p}}\right|=e^{-i \phi S_{z}} e^{-i \theta S_{y}} \hat{e}_{m} \hat{e}_{m}^{T} e^{i \theta S_{y}} e^{i \phi S_{z}}
$$

determine the microscopic form of the scattering rates. Specifically, we here review the simplest cases of $S \leq 3 / 2$. For the $S=1 / 2$ case the result is well known

$$
\hat{P}_{m, \boldsymbol{p}}=\frac{1+4 m(\hat{d} \cdot \boldsymbol{S})}{2} .
$$

In contrast, for the $S=1$ one finds instead a different expression

$$
\hat{P}_{m, \boldsymbol{p}}=\left(1-m^{2}\right)+\frac{m}{2}(\hat{d} \cdot \boldsymbol{S})+\frac{3 m^{2}-2}{2}(\hat{d} \cdot \boldsymbol{S})^{2} .
$$

Finally, for the $S=3 / 2$ situation one needs to distinguish between projections with $|m|=3 / 2$

$$
\hat{P}_{m, \boldsymbol{p}}=-\frac{1}{16}-\frac{m}{36}(\hat{d} \cdot \boldsymbol{S})+\frac{1}{4}(\hat{d} \cdot \boldsymbol{S})^{2}+\frac{m}{9}(\hat{d} \cdot \boldsymbol{S})^{3},
$$

and alternatively with $|m|=1 / 2$, for which

$$
\hat{P}_{m, \boldsymbol{p}}=\frac{9}{16}+\frac{9 m}{4} \hat{d} \cdot \boldsymbol{S}-\frac{1}{4}(\hat{d} \cdot \boldsymbol{S})^{2}-m(\hat{d} \cdot \boldsymbol{S})^{3} .
$$

\subsection{Overlap of states}

The overlap of states in the same band can be directly calculated in complete analogy to projectors. For the above specified model examples they look as follows. For $S=1 / 2$

$$
\left|\left\langle u_{m, \boldsymbol{p}_{1}} \mid u_{m, \boldsymbol{p}_{2}}\right\rangle\right|^{2}=\frac{1+\hat{d}_{1} \cdot \hat{d}_{2}}{2} .
$$

For the $S=1$ case the overlap depends on the value of $m$. The simplest is that of $m=0$

$$
\left|\left\langle u_{m, p_{1}} \mid u_{m, p_{2}}\right\rangle\right|^{2}=\left(\hat{d}_{1} \cdot \hat{d}_{2}\right)^{2},
$$


whereas $|m|=1$ is given by

$$
\left|\left\langle u_{m, p_{1}} \mid u_{m, p_{2}}\right\rangle\right|^{2}=\frac{\left(1+\hat{d}_{1} \cdot \hat{d}_{2}\right)^{2}}{4} .
$$

The overlap for $S=3 / 2$ with $|m|=1 / 2$ is more involved

$$
\left|\left\langle u_{m, \boldsymbol{p}_{1}} \mid u_{m, \boldsymbol{p}_{2}}\right\rangle\right|^{2}=\frac{\left(1+\hat{d}_{1} \cdot \hat{d}_{2}\right)^{3}}{8}-\left(\hat{d}_{1} \cdot \hat{d}_{2}\right)\left(\hat{d}_{1} \times \hat{d}_{2}\right)^{2},
$$

while the last one for $|m|=3 / 2$ is

$$
\left|\left\langle u_{m, \boldsymbol{p}_{1}} \mid u_{m, \boldsymbol{p}_{2}}\right\rangle\right|^{2}=\frac{\left(1+\hat{d}_{1} \cdot \hat{d}_{2}\right)^{3}}{8}
$$

In all the above expressions we used a shorthand notation $\hat{d}_{i}=\boldsymbol{d}\left(\boldsymbol{p}_{i}\right) / d\left(\boldsymbol{p}_{i}\right)$.

\subsection{Pancharatnam phase}

The Pancharatnam phase $\Phi_{p_{1} p_{2} p_{3}}$ of the underlying electronic band structure is defined by the Bloch state overlap of the form

$$
\Phi_{\boldsymbol{p}_{1} \boldsymbol{p}_{2} \boldsymbol{p}_{3}}=\arg \left[\left\langle u_{\boldsymbol{p}_{1}} \mid u_{\boldsymbol{p}_{2}}\right\rangle\left\langle u_{\boldsymbol{p}_{2}} \mid u_{\boldsymbol{p}_{3}}\right\rangle\left\langle u_{\boldsymbol{p}_{3}} \mid u_{\boldsymbol{p}_{1}}\right\rangle\right] .
$$

Interestingly, as alluded in the introduction, there is a limit when both the skew scattering rate and the side jump contribution can be directly related to $\Phi_{p_{1}} p_{2} p_{3}$. Indeed, as it follows from the collision terms derived in the preceding sections, the antisymmetric part of the scattering rate depends on both properties of band structure and local impurity potential. Thus generic calculations are possible only based on first principle numerical methods. However, under the simplifying assumptions of a single relevant band and a smooth centrosymmetric potential, we demonstrated that skew scattering depends on the quantitiy $Z_{\boldsymbol{p}_{1} \boldsymbol{p}_{2} \boldsymbol{p}_{3}}$, Eq. (2.24d) (we use $l_{i}=\left(n, \boldsymbol{p}_{i}\right), i=1,2,3$ and suppress the band index $n$ ) which is non-zero only if the Pancharatnam phase in Eq. (4.14) is finite. Below we report results for $Z_{p_{1} p_{2} p_{3}}$ where we retained only intraband contributions. For $S=1 / 2$ the result was derived earlier [77]

$$
Z_{p_{1} \boldsymbol{p}_{2} \boldsymbol{p}_{3}}=\frac{m}{2} \hat{d}_{1} \cdot\left(\hat{d}_{2} \times \hat{d}_{3}\right)
$$

For $S=1$ we find

$$
Z_{p_{1} p_{2} p_{2}}=\frac{m}{8} \hat{d}_{1} \cdot\left(\hat{d}_{2} \times \hat{d}_{3}\right)\left(1+\hat{d}_{1} \cdot \hat{d}_{2}+\hat{d}_{2} \cdot \hat{d}_{3}+\hat{d}_{3} \cdot \hat{d}_{1}\right)
$$

For $S=3 / 2$ the expressions we get are rather cumbersome

$$
\begin{aligned}
Z_{p_{1} p_{2} p_{3}} & = \pm \frac{\hat{d}_{1} \cdot\left(\hat{d}_{2} \times \hat{d}_{3}\right)}{128}\left[13+12\left(\hat{d}_{1} \cdot \hat{d}_{2}+\circlearrowright\right)-\left[\left(\hat{d}_{2} \cdot \hat{d}_{3}\right)^{2}+\circlearrowright\right]\right. \\
& \left.+8\left(\hat{d}_{1} \cdot \hat{d}_{3} \hat{d}_{2} \cdot d_{3}+\circlearrowright\right)+14\left(\hat{d}_{1} \cdot \hat{d}_{2} \hat{d}_{2} \cdot \hat{d}_{3} \hat{d}_{3} \cdot \hat{d}_{1}\right)-9\left(\hat{d}_{1} \cdot\left(\hat{d}_{2} \times \hat{d}_{3}\right)\right)^{2}\right]
\end{aligned}
$$

for $|m|=3 / 2$ with symbol $\circlearrowright$ indicating cyclic permutation of indices $1 \rightarrow 2 \rightarrow 3 \rightarrow 1$ in the sum of respective terms. Lastly, when $|m|=1 / 2$ the result is

$$
\begin{aligned}
Z_{p_{1} p_{2} p_{3}} & = \pm \frac{\hat{d}_{1} \cdot\left(\hat{d}_{2} \times \hat{d}_{3}\right)}{128}\left[-247+12\left(\hat{d}_{1} \cdot \hat{d}_{2}+\circlearrowright\right)+243\left[\left(\hat{d}_{2} \cdot \hat{d}_{3}\right)^{2}+\circlearrowright\right]\right. \\
& \left.-36\left(\hat{d}_{1} \cdot \hat{d}_{3} \hat{d}_{2} \cdot d_{3}+\circlearrowright\right)-378\left(\hat{d}_{1} \cdot \hat{d}_{2} \hat{d}_{2} \cdot \hat{d}_{3} \hat{d}_{3} \cdot \hat{d}_{1}\right)+243\left(\hat{d}_{1} \cdot\left(\hat{d}_{2} \times \hat{d}_{3}\right)\right)^{2}\right] .
\end{aligned}
$$

These formulas give us all the required ingredients to calculate anomalous Hall conductance. We next proceed to solve the kinetic equation in the exemplary case of $S=1$. 


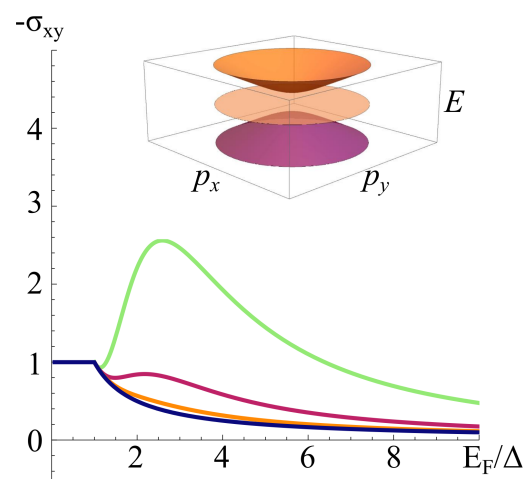

b

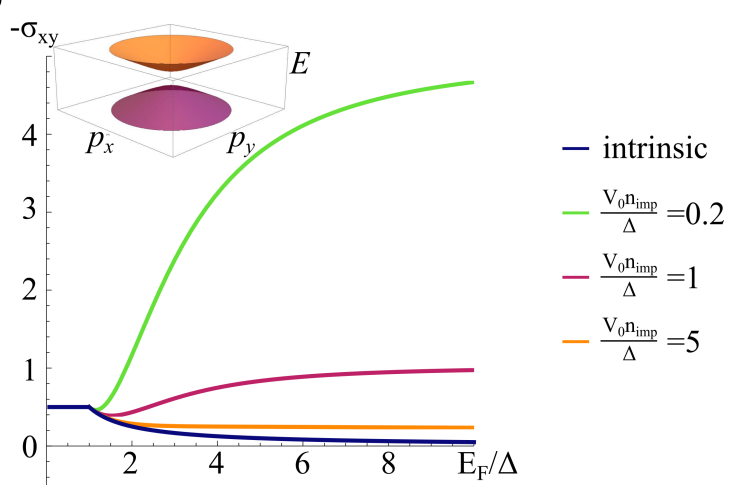

Figure 5: a Anomalous Hall conductivity of massive spin-1 fermions plotted from Eq. (4.19). The inset shows the low-energy band structure dispersion of a single massive pseudospin-1 fermion. b Anomalous Hall effect of massive 2D Dirac fermions, Eq. (4.21). The inset shows dispersion of the model.

\subsection{AHE of pseudospin-1 fermions}

To illustrate the findings of the previous section, we calculate the anomalous Hall response from Eq. (1.1) for $S=1, d_{0}(\boldsymbol{p})=0$ and $\boldsymbol{d}(\boldsymbol{p})=\left(v p_{x}, v p_{y}, \Delta\right)$ in a two dimensional system with a single spin-1 touching point. This can be modelled on a Kagome lattice with an appropriately tuned flux pattern $(\Delta \neq 0$ when the fluxes are not in $\pi \mathbb{Z}$ ), see Ref. [98] for details. It is worthwhile to highlight that a single spin-1 node may appear in the Brillouin zone without defying the fermion-doubling theorem.

We concentrate on $m=+1$ and take $f=f_{0}+\boldsymbol{p} \cdot \boldsymbol{g}$, so that the collision integral is

$$
\operatorname{St}_{\text {Born }}\{f\}+\operatorname{St}_{\mathrm{sk}}\{f\}=-\frac{\boldsymbol{p} \cdot \boldsymbol{g}}{\tau}-\frac{\hat{e}_{z} \cdot[\boldsymbol{p} \times \boldsymbol{g}]}{\tau_{\mathrm{sk}}},
$$

where

$$
\frac{1}{\tau}=2 \pi n_{\mathrm{imp}} V_{0}^{2} \nu \frac{E^{4}+2 \Delta^{2} E^{2}+5 \Delta^{4}}{8 E^{4}}, \quad \frac{1}{\tau_{\mathrm{sk}}}=(2 \pi)^{2} n_{\mathrm{imp}} V_{0}^{3} \nu^{2} \frac{\Delta^{3}\left(E^{2}-\Delta^{2}\right)}{4 E^{5}} .
$$

Here we used Eqs. (4.11), (4.15b) in the evaluation of Eq. (2.27) and assumed point like impurities of strength $V_{0}$ (for details see Appendix A), the density of states is denoted $\nu=\nu(E)=\theta(E-\Delta) E /\left(2 \pi v^{2}\right)$. The solution of the Boltzmann equation to leading order in external static field leads to

$$
\boldsymbol{g}=\frac{v^{2}}{E_{\boldsymbol{p}}}\left(\begin{array}{cc}
1 & -\tau / \tau_{\mathrm{sk}} \\
\tau / \tau_{\mathrm{sk}} & 1
\end{array}\right) \tau e \mathcal{E} \delta\left(E_{F}-E_{\boldsymbol{p}}\right)
$$

so that $\sigma_{x y}=\sigma_{x y}^{\text {int }}+\sigma_{x y}^{\text {sk }}$ with respective terms

$$
\begin{aligned}
& \sigma_{x y}^{\mathrm{int}}=-e^{2} \int_{p} f_{0} \Omega_{z}=-\frac{e^{2}}{2 \pi \hbar} \frac{\Delta}{E_{F}} \\
& \sigma_{x y}^{\mathrm{sk}}=-e^{2} \int_{p} \delta\left(E_{p}-E_{F}\right) \frac{v^{4} p^{2}}{E_{p}^{2}} \frac{\tau^{2}}{2 \tau_{\mathrm{sk}}}=-\frac{e^{2}}{\hbar}(\Delta \tau)\left(\nu V_{0}\right) \frac{\Delta^{2}\left(E_{F}^{2}-\Delta^{2}\right)^{2}}{E_{F}^{2}\left(E_{F}^{4}+2 \Delta^{2} E_{F}^{2}+5 \Delta^{4}\right)} .
\end{aligned}
$$

In the final result we restored Planck's constant $\hbar=h / 2 \pi$. The relative importance of the side-jump contribution can be also estimated $\sigma_{x y}^{\mathrm{sk}} / \sigma_{x y}^{\mathrm{sj}} \sim\left(\nu V_{0}\right)\left(E_{F} \tau\right)$. For moderately strong impurity potential when, $\nu V_{0} \sim 1$, skew scattering dominates in the metallic regime $E_{F} \tau \gg 1$. Note that the flat $m=0$ band does not contribute, since it's Berry curvature vanishes and it moreover does not intersect the Fermi surface.

Perhaps most notably, the skew scattering contribution to the anomalous Hall response of pseudospin1 fermions, Fig. 5 a, results in a non-monotonic behavior and decays as $\Delta^{2} / E_{F}^{2}$ at large Fermi energies. This should be contrasted to the more familiar behavior of ordinary spin- $1 / 2$ fermions [58], governed by 
Eq. (1.1) for $S=1 / 2$ and $d_{0}(\boldsymbol{p})=0$ and $\boldsymbol{d}(\boldsymbol{p})=2\left(v p_{x}, v p_{y}, \Delta\right)$ (this model has the same density of states $\nu=\nu(E)=\theta(E-\Delta) E /\left(2 \pi v^{2}\right)$ as the spin-1 model). In that case we thereby have

$$
\frac{1}{\tau}=2 \pi n_{\mathrm{imp}} V_{0}^{2} \nu \frac{E^{2}+3 \Delta^{2}}{4 E^{2}}, \quad \frac{1}{\tau_{\mathrm{sk}}}=(2 \pi)^{2} n_{\mathrm{imp}} V_{0}^{3} \nu^{2} \frac{\Delta\left(E^{2}-\Delta^{2}\right)}{8 E^{3}},
$$

so that respective transverse conductivity contributions are given by

$$
\sigma_{x y}^{\text {int }}=-\frac{e^{2}}{2 \pi \hbar} \frac{\Delta}{2 E_{F}}, \quad \sigma_{x y}^{\text {sk }}=-\frac{e^{2}}{\hbar}(\Delta \tau)\left(\nu V_{0}\right) \frac{\left(E_{F}^{2}-\Delta^{2}\right)^{2}}{4 E_{F}^{2}\left(E_{F}^{2}+3 \Delta^{2}\right)} .
$$

Note that, in contrast to Eq. (4.19), here the skew scattering contribution saturates at large energies. This crucial distinction of multifold fermions as compared to ordinary spin- $1 / 2$ fermions, which is further exemplified in Fig. 5 b, is a consequence of the differences in the Pancharatnam phase, Eqs. (4.14), and ultimately a consequence of a different behavior of wave function overlaps.

\section{Summary and Outlook}

To conclude, we have presented a systematic derivation of the Boltzmann equation for materials with finite Berry curvature in the regime of small frequency, Eq. (2.27), and large frequency, Eq. (2.32). Our derivation is valid up to second order in driving fields and included a careful treatment of quantum scattering events, such as side jump, skew scattering and shift current contributions. Our results thus serve as the foundation for the theoretical description of linear and nonlinear transport and optics phenomena in topological quantum materials. As an application of our theory, we have considered the anomalous Hall response in multifold fermion systems. Specifically, we have derived the formulae for skew scattering probability, Eqs. (2.24), (4.15) which determine the skew scattering for spin- 1 and spin-3/2 multifold fermions. As a concrete illustration, we derived the anomalous Hall response for a simple, isotropic model of gapped spin-1 fermions in two dimensions, Eq. (4.19), see Fig. 5.

Directions of future research include further studies of inelastic skew scattering and side jump contributions due to electron-electron interaction [107] in the hydrodynamic regime or electron-phonon interaction [108]. It should be possible to readily incorporated those using a similar diagrammatic technique as exposed in Fig. 3. Moreover, a careful study of the interplay of mixed contributions of photon and impurity scattering for a concrete model is left for the future as well as the generalization of the Boltzmann-Berry equation to non-Abelian Berry curvature. Finally, in regards of multifold fermion systems, which are at the center of the attention for the quantized circular photogalvanic effect, a careful investigation of photocurrent relaxation is of experimental and theoretical interest and readily achievable within the presented formalism.

\section{Acknowledgments}

We thank M. Dzero, S. Li, P. Ostrovsky, D. Pesin, H.-Y. Xie for fruitful discussions and prior collaboration on related topics that inspired this study. We are also grateful to Zongzheng Du for communicating to us on the progress and remaining open question with the diagrammatic calculations of the nonlinear Hall effect. The work of A.L. at UW-Madison was supported by the National Science Foundation CAREER Grant No. DMR-1653661.

\section{Appendix A. Collision integrals and averages}

In this section of the appendix we present supplemental details regarding calculations carried out in Sec. 4.5. For the point like impurities of strength $V_{0}$ and the relevant collision integrals that determine intrinsic and skew scattering contributions are given by

$$
\begin{aligned}
\mathrm{St}_{\mathrm{Born}}^{[2]}\{f\} & =-2 \pi n_{\mathrm{imp}} V_{0}^{2} \int_{\boldsymbol{p}^{\prime}} \delta\left(E_{\boldsymbol{p}}-E_{\boldsymbol{p}^{\prime}}\right)\left|\left\langle u_{m, \boldsymbol{p}} \mid u_{m, \boldsymbol{p}^{\prime}}\right\rangle\right|^{2}\left[f(\boldsymbol{p})-f\left(\boldsymbol{p}^{\prime}\right)\right], \\
\mathrm{St}_{\mathrm{sk}}^{[3 a]}\{f\} & =-2 \pi n_{\mathrm{imp}} V_{0}^{3} \int_{\boldsymbol{p}^{\prime} \boldsymbol{p}^{\prime \prime}} \delta\left(E_{\boldsymbol{p}}-E_{\boldsymbol{p}^{\prime}}\right) \delta\left(E_{\boldsymbol{p}}-E_{\boldsymbol{p}^{\prime \prime}}\right) Z_{\boldsymbol{p} \boldsymbol{p}^{\prime} \boldsymbol{p}^{\prime \prime}} f\left(\boldsymbol{p}^{\prime}\right) .
\end{aligned}
$$


In order to extract scattering times $\tau$ and $\tau_{\text {sk }}$ entering Eq. (4.16) we need to perform several averages over the Fermi surface. These averages can be split in groups of scalars

$$
\left\langle\hat{d} \cdot \hat{d}^{\prime}\right\rangle_{\hat{p}^{\prime}}=\frac{\Delta^{2}}{E^{2}}, \quad\left\langle\left(\hat{d} \cdot \hat{d}^{\prime}\right)^{2}\right\rangle_{\hat{p}^{\prime}}=\frac{E^{4}-2 E^{2} \Delta^{2}+3 \Delta^{4}}{2 E^{4}}
$$

vectors

$$
\left\langle\boldsymbol{p}^{\prime}\left(\hat{d} \cdot \hat{d}^{\prime}\right)\right\rangle_{\hat{p}^{\prime}}=\frac{E^{2}-\Delta^{2}}{2 E^{2}} \boldsymbol{p}, \quad\left\langle\boldsymbol{p}^{\prime}\left(\hat{d} \cdot \hat{d}^{\prime}\right)^{2}\right\rangle_{\hat{p}^{\prime}}=\frac{\Delta^{2}\left(E^{2}-\Delta^{2}\right)}{E^{4}} \boldsymbol{p},
$$

and tensors

$$
\begin{aligned}
\left\langle\boldsymbol{p}^{\prime} \cdot \boldsymbol{g} \hat{d} \cdot\left(\hat{d}^{\prime} \times \hat{d}^{\prime \prime}\right)\right\rangle_{\hat{p}^{\prime}, \hat{p}^{\prime \prime}}=\frac{\Delta\left(E^{2}-\Delta^{2}\right)}{2 E^{3}} \hat{e}_{z} \cdot(\boldsymbol{p} \times \boldsymbol{g}), \\
\left\langle\boldsymbol{p}^{\prime} \cdot \boldsymbol{g} \hat{d} \cdot\left(\hat{d}^{\prime} \times \hat{d}^{\prime \prime}\right)\left(\hat{d} \cdot \hat{d}^{\prime}\right)\right\rangle_{\hat{p}^{\prime}, \hat{p}^{\prime \prime}}=\frac{\Delta^{2}}{E^{2}}\left\langle\boldsymbol{p}^{\prime} \cdot \boldsymbol{g} \hat{d} \cdot\left(\hat{d}^{\prime} \times \hat{d}^{\prime \prime}\right)\right\rangle_{\hat{p}^{\prime}, \hat{p}^{\prime \prime}}, \\
\left\langle\boldsymbol{p}^{\prime} \cdot \boldsymbol{g} \hat{d} \cdot\left(\hat{d}^{\prime} \times \hat{d}^{\prime \prime}\right)\left(\hat{d}^{\prime} \cdot \hat{d}^{\prime \prime}\right)\right\rangle_{\hat{p}^{\prime}, \hat{p}^{\prime \prime}}=\frac{3 \Delta^{2}-E^{2}}{2 E^{2}}\left\langle\boldsymbol{p}^{\prime} \cdot \boldsymbol{g} \hat{d} \cdot\left(\hat{d}^{\prime} \times \hat{d}^{\prime \prime}\right)\right\rangle_{\hat{p}^{\prime}, \hat{p}^{\prime \prime}} \\
\left\langle\boldsymbol{p}^{\prime} \cdot \boldsymbol{g} \hat{d} \cdot\left(\hat{d}^{\prime} \times \hat{d}^{\prime \prime}\right)\left(\hat{d} \cdot \hat{d}^{\prime \prime}\right)\right\rangle_{\hat{p}^{\prime}, \hat{p}^{\prime \prime}}=\frac{3 \Delta^{2}-E^{2}}{2 E^{2}}\left\langle\boldsymbol{p}^{\prime} \cdot \boldsymbol{g} \hat{d} \cdot\left(\hat{d}^{\prime} \times \hat{d}^{\prime \prime}\right)\right\rangle_{\hat{p}^{\prime}, \hat{p}^{\prime \prime}}
\end{aligned}
$$

\section{References}

[1] N. Nagaosa, J. Sinova, S. Onoda, A. H. MacDonald, N. P. Ong, Anomalous hall effect, Rev. Mod. Phys. 82 (2010) 1539-1592. doi:10.1103/RevModPhys.82.1539. URL https://link.aps.org/doi/10.1103/RevModPhys.82.1539

[2] D. Xiao, M.-C. Chang, Q. Niu, Berry phase effects on electronic properties, Rev. Mod. Phys. 82 (2010) $1959-2007$. doi:10.1103/RevModPhys.82.1959.

URL https://link.aps.org/doi/10.1103/RevModPhys.82.1959

[3] J. Maciejko, T. L. Hughes, S.-C. Zhang, The quantum spin hall effect, Annual Review of Condensed Matter Physics 2 (1) (2011) 31-53. doi:10.1146/annurev-conmatphys-062910-140538.

URL https://doi.org/10.1146/annurev-conmatphys-062910-140538

[4] J. Sinova, S. O. Valenzuela, J. Wunderlich, C. H. Back, T. Jungwirth, Spin hall effects, Rev. Mod. Phys. 87 (2015) 1213-1260. doi:10.1103/RevModPhys.87.1213. URL https://link.aps.org/doi/10.1103/RevModPhys.87.1213

[5] C.-X. Liu, S.-C. Zhang, X.-L. Qi, The quantum anomalous hall effect: Theory and experiment, Annual Review of Condensed Matter Physics 7 (1) (2016) 301-321. doi:10.1146/annurev-conmatphys-031115-011417. URL https://doi.org/10.1146/annurev-conmatphys-031115-011417

[6] Y. Ren, Z. Qiao, Q. Niu, Topological phases in two-dimensional materials: a review, Reports on Progress in Physics 79 (6) (2016) 066501. doi:10.1088/0034-4885/79/6/066501.

[7] P. W. Anderson, Hall effect in the two-dimensional luttinger liquid, Phys. Rev. Lett. 67 (1991) 2092-2094. doi:10.1103/PhysRevLett.67.2092.

URL https://link.aps.org/doi/10.1103/PhysRevLett.67.2092

[8] N. P. Ong, P. W. Anderson, Comment on "anomalous hall effect in yba $\mathrm{cu}_{3} \mathrm{O} 7$ ", Phys. Rev. Lett. 78 (1997) $977-977$. doi:10.1103/PhysRevLett.78.977. URL https://link.aps.org/doi/10.1103/PhysRevLett.78.977

[9] Y. Zhang, N. P. Ong, P. W. Anderson, D. A. Bonn, R. Liang, W. N. Hardy, Giant enhancement of the thermal hall conductivity $\kappa_{x y}$ in the superconductor yba $\mathrm{Cu}_{3} \mathrm{O}_{7}$, Phys. Rev. Lett. 86 (2001) 890-893. doi:10.1103/PhysRevLett.86.890. URL https://link.aps.org/doi/10.1103/PhysRevLett.86.890

[10] J. Kondo, Anomalous hall effect and magnetoresistance of ferromagnetic metals, Progress of Theoretical Physics 27 (4) (1962) 772-792.

[11] P. Coleman, P. W. Anderson, T. V. Ramakrishnan, Theory for the anomalous hall constant of mixed-valence systems, Phys. Rev. Lett. 55 (1985) 414-417. doi:10.1103/PhysRevLett.55.414. URL https://link.aps.org/doi/10.1103/PhysRevLett.55.414

[12] T. Ramakrishnan, P. Coleman, P. Anderson, Anomalous hall effect in kondo and mixed valent systems, Journal of magnetism and magnetic materials 47 (1985) 493-496.

[13] A. Kapitulnik, J. Xia, E. Schemm, A. Palevski, Polar kerr effect as probe for time-reversal symmetry breaking in unconventional superconductors, New Journal of Physics 11 (5) (2009) 055060.

[14] P. W. Anderson, P. Morel, Generalized bardeen-cooper-schrieffer states and the proposed low-temperature phase of liquid he ${ }^{3}$, Phys. Rev. 123 (1961) 1911-1934. doi:10.1103/PhysRev.123.1911.

URL https://link.aps.org/doi/10.1103/PhysRev.123.1911 
[15] P. W. Anderson, W. F. Brinkman, Anisotropic superfluidity in ${ }^{3}$ He: A possible interpretation of its stability as a spinfluctuation effect, Phys. Rev. Lett. 30 (1973) 1108-1111. doi:10.1103/PhysRevLett.30.1108.

URL https://link.aps.org/doi/10.1103/PhysRevLett.30.1108

[16] R. Karplus, J. M. Luttinger, Hall effect in ferromagnetics, Phys. Rev. 95 (1954) 1154-1160. doi:10.1103/PhysRev.95.1154. URL https://link.aps.org/doi/10.1103/PhysRev.95.1154

[17] J. Smit, The spontaneous hall effect in ferromagnetics i, Physica 21 (6) (1955) 877. doi:https://doi.org/10.1016/S00318914(55)92596-9. URL http://www.sciencedirect.com/science/article/pii/S0031891455925969

[18] J. Smit, The spontaneous hall effect in ferromagnetics ii, Physica 24 (1) (1958) 39. doi:https://doi.org/10.1016/S00318914(58)93541-9. URL http://www.sciencedirect.com/science/article/pii/S0031891458935419

[19] L. Berger, Side-jump mechanism for the hall effect of ferromagnets, Phys. Rev. B 2 (1970) $4559-4566$. doi:10.1103/PhysRevB.2.4559.

[20] M. Dyakonov, V. Perel, Possibility of orienting electron spins with current, JETP Letters 13 (11) (1971) 467.

[21] M. Dyakonov, V. Perel, Current-induced spin orientation of electrons in semiconductors, Physics Letters A 35 (6) (1971) 459 - 460. doi:https://doi.org/10.1016/0375-9601(71)90196-4. URL http://www.sciencedirect.com/science/article/pii/0375960171901964

[22] K. v. Klitzing, G. Dorda, M. Pepper, New method for high-accuracy determination of the fine-structure constant based on quantized hall resistance, Phys. Rev. Lett. 45 (1980) 494-497. doi:10.1103/PhysRevLett.45.494. URL https://link.aps.org/doi/10.1103/PhysRevLett.45.494

[23] D. J. Thouless, M. Kohmoto, M. P. Nightingale, M. den Nijs, Quantized hall conductance in a two-dimensional periodic potential, Phys. Rev. Lett. 49 (1982) 405-408. doi:10.1103/PhysRevLett.49.405. URL https://link.aps.org/doi/10.1103/PhysRevLett.49.405

[24] F. D. M. Haldane, Model for a quantum hall effect without landau levels: Condensed-matter realization of the "parity anomaly", Phys. Rev. Lett. 61 (1988) 2015-2018. doi:10.1103/PhysRevLett.61.2015. URL https://link.aps.org/doi/10.1103/PhysRevLett.61.2015

[25] M. V. Berry, Quantal phase factors accompanying adiabatic changes, Proc. R. Soc. Lond. A 392 (1984) 45.

[26] M.-C. Chang, Q. Niu, Berry phase, hyperorbits, and the hofstadter spectrum, Phys. Rev. Lett. 75 (1995) $1348-1351$. doi:10.1103/PhysRevLett.75.1348.

URL https://link.aps.org/doi/10.1103/PhysRevLett.75.1348

[27] S. Pancharatnam, Generalized theory of interference and its applications, Proc. Indian Acad. Sci. 44 (1956) 247.

[28] V. Belinicher, E. Ivchenko, B. Sturman, Kinetic theory of the displacement photovoltaic effect in piezoelectrics, Zh. Eksp. Teor. Fiz. 83 (1982) 649-661.

[29] N. A. Sinitsyn, Q. Niu, A. H. MacDonald, Coordinate shift in the semiclassical boltzmann equation and the anomalous hall effect, Phys. Rev. B 73 (2006) 075318. doi:10.1103/PhysRevB.73.075318. URL https://link.aps.org/doi/10.1103/PhysRevB.73.075318

[30] C. L. Kane, E. J. Mele, $Z_{2}$ topological order and the quantum spin hall effect, Phys. Rev. Lett. 95 (2005) 146802. doi:10.1103/PhysRevLett.95.146802. URL https://link.aps.org/doi/10.1103/PhysRevLett.95.146802

[31] B. A. Bernevig, T. L. Hughes, S.-C. Zhang, Quantum spin hall effect and topological phase transition in hgte quantum wells, Science 314 (5806) (2006) 1757-1761. doi:10.1126/science.1133734.

[32] M. König, S. Wiedmann, C. Brüne, A. Roth, H. Buhmann, L. W. Molenkamp, X.-L. Qi, S.-C. Zhang, Quantum spin hall insulator state in hgte quantum wells, Science 318 (5851) (2007) 766-770. doi:10.1126/science.1148047.

[33] C.-Z. Chang, J. Zhang, X. Feng, J. Shen, Z. Zhang, M. Guo, K. Li, Y. Ou, P. Wei, L.-L. Wang, Z.-Q. Ji, Y. Feng, S. Ji, X. Chen, J. Jia, X. Dai, Z. Fang, S.-C. Zhang, K. He, Y. Wang, L. Lu, X.-C. Ma, Q.-K. Xue, Experimental observation of the quantum anomalous hall effect in a magnetic topological insulator, Science 340 (6129) (2013) $167-170$. doi:10.1126/science.1234414.

[34] K. Komatsu, Y. Morita, E. Watanabe, D. Tsuya, K. Watanabe, T. Taniguchi, S. Moriyama, Observation of the quantum valley hall state in ballistic graphene superlattices, Science Advances 4 (5). doi:10.1126/sciadv.aaq0194.

[35] A. L. Sharpe, E. J. Fox, A. W. Barnard, J. Finney, K. Watanabe, T. Taniguchi, M. A. Kastner, D. Goldhaber-Gordon, Emergent ferromagnetism near three-quarters filling in twisted bilayer graphene, Science 365 (6453) (2019) 605-608. doi:10.1126/science.aaw3780.

[36] A. Langenfeld, P. Wölfle, Absence of quantum corrections to the anomalous hall conductivity, Phys. Rev. Lett. 67 (1991) 739-741. doi:10.1103/PhysRevLett.67.739. URL https://link.aps.org/doi/10.1103/PhysRevLett.67.739

[37] K. A. Muttalib, P. Wölfle, Disorder and temperature dependence of the anomalous hall effect in thin ferromagnetic films: Microscopic model, Phys. Rev. B 76 (2007) 214415. doi:10.1103/PhysRevB.76.214415. URL https://link.aps.org/doi/10.1103/PhysRevB.76.214415

[38] S. M. Badalyan, G. Vignale, Spin hall drag in electronic bilayers, Phys. Rev. Lett. 103 (2009) 196601. doi:10.1103/PhysRevLett.103.196601. URL https://link.aps.org/doi/10.1103/PhysRevLett.103.196601

[39] S. Li, A. Levchenko, Temperature dependence of the anomalous hall effect from electron interactions, Phys. Rev. Lett. 124 (2020) 156802. doi:10.1103/PhysRevLett.124.156802. URL https://link.aps.org/doi/10.1103/PhysRevLett.124.156802

[40] A. Avdoshkin, V. Kozii, J. E. Moore, Interactions remove the quantization of the chiral photocurrent at weyl points, 
Phys. Rev. Lett. 124 (2020) 196603. doi:10.1103/PhysRevLett.124.196603.

URL https://link.aps.org/doi/10.1103/PhysRevLett.124.196603

[41] I. Mandal, Effect of interactions on the quantization of the chiral photocurrent for double-weyl semimetals, Symmetry 12 (6) (2020) 919.

[42] V. K. Dugaev, A. Crépieux, P. Bruno, Localization corrections to the anomalous hall effect in a ferromagnet, Phys. Rev. B 64 (2001) 104411. doi:10.1103/PhysRevB.64.104411.

URL https://link.aps.org/doi/10.1103/PhysRevB.64.104411

[43] P. Wölfle, K. Muttalib, Anomalous hall effect in ferromagnetic disordered metals, Annalen der Physik 15 (7-8) (2006) 508-519. doi:https://doi.org/10.1002/andp.200510206.

[44] H. Meier, M. Y. Kharitonov, K. B. Efetov, Anomalous hall effect in granular ferromagnetic metals and effects of weak localization, Phys. Rev. B 80 (2009) 045122. doi:10.1103/PhysRevB.80.045122.

URL https://link.aps.org/doi/10.1103/PhysRevB.80.045122

[45] E. J. König, P. M. Ostrovsky, I. V. Protopopov, I. V. Gornyi, I. S. Burmistrov, A. D. Mirlin, Half-integer quantum hall effect of disordered dirac fermions at a topological insulator surface, Phys. Rev. B 90 (2014) 165435. doi:10.1103/PhysRevB.90.165435.

URL https://link.aps.org/doi/10.1103/PhysRevB.90.165435

[46] J. M. Luttinger, Theory of the hall effect in ferromagnetic substances, Phys. Rev. 112 (1958) $739-751$. doi:10.1103/PhysRev.112.739.

URL https://link.aps.org/doi/10.1103/PhysRev.112.739

[47] E. Adams, E. Blount, Energy bands in the presence of an external force field—ii: Anomalous velocities, Journal of Physics and Chemistry of Solids 10 (4) (1959) 286 - 303. doi:https://doi.org/10.1016/0022-3697(59)90004-6. URL http://www.sciencedirect.com/science/article/pii/0022369759900046

[48] E. I. Blount, Formalisms of Band Theory, Solid State Physics - Advances in Research and Applications 13 (1962) $305-373$.

[49] J.-N. Chazalviel, Spin-dependent hall effect in semiconductors, Phys. Rev. B 11 (1975) $3918-3934$. doi:10.1103/PhysRevB.11.3918.

URL https://link.aps.org/doi/10.1103/PhysRevB.11.3918

[50] Nozières, P., Lewiner, C., A simple theory of the anomalous hall effect in semiconductors, J. Phys. France 34 (10) (1973) 901-915. doi:10.1051/jphys:019730034010090100. URL https://doi.org/10.1051/jphys:019730034010090100

[51] S. K. Lyo, T. Holstein, Side-jump mechanism for ferromagnetic hall effect, Phys. Rev. Lett. 29 (1972) $423-425$. doi:10.1103/PhysRevLett.29.423.

URL https://link.aps.org/doi/10.1103/PhysRevLett.29.423

[52] C. Gorini, P. Schwab, R. Raimondi, A. L. Shelankov, Non-abelian gauge fields in the gradient expansion: Generalized boltzmann and eilenberger equations, Phys. Rev. B 82 (2010) 195316. doi:10.1103/PhysRevB.82.195316.

URL https://link.aps.org/doi/10.1103/PhysRevB.82.195316

[53] C. H. Wong, Y. Tserkovnyak, Quantum kinetic equation in phase-space textured multiband systems, Phys. Rev. B 84 (2011) 115209. doi:10.1103/PhysRevB.84.115209. URL https://link.aps.org/doi/10.1103/PhysRevB.84.115209

[54] C. Wickles, W. Belzig, Effective quantum theories for Bloch dynamics in inhomogeneous systems with nontrivial band structure, Physical Review B - Condensed Matter and Materials Physics 88 (4) (2013) 1-18.

[55] A. J. S. Bolet, Berry Curvature as a Multi-Band Effect in Boltzmann Equations, Masters thesis at the Niels Bohr Institute, University of Copenhagen, Denmark, 2014.

[56] A. Sekine, D. Culcer, A. H. MacDonald, Quantum kinetic theory of the chiral anomaly, Phys. Rev. B 96 (2017) 235134. doi:10.1103/PhysRevB.96.235134. URL https://link.aps.org/doi/10.1103/PhysRevB.96.235134

[57] N. A. Sinitsyn, Semiclassical theories of the anomalous hall effect, Journal of Physics: Condensed Matter 20 (2) (2007) 023201. doi:10.1088/0953-8984/20/02/023201. URL https://doi.org/10.1088/0953-8984/20/02/023201

[58] N. A. Sinitsyn, A. H. MacDonald, T. Jungwirth, V. K. Dugaev, J. Sinova, Anomalous hall effect in a two-dimensional dirac band: The link between the kubo-streda formula and the semiclassical boltzmann equation approach, Phys. Rev. B 75 (2007) 045315. doi:10.1103/PhysRevB.75.045315. URL https://link.aps.org/doi/10.1103/PhysRevB.75.045315

[59] A. A. Kovalev, K. Výborný, J. Sinova, Hybrid skew scattering regime of the anomalous hall effect in rashba systems: Unifying keldysh, boltzmann, and kubo formalisms, Phys. Rev. B 78 (2008) 041305. doi:10.1103/PhysRevB.78.041305. URL https://link.aps.org/doi/10.1103/PhysRevB.78.041305

[60] J. Mitscherling, Longitudinal and anomalous hall conductivity of a general two-band model, Phys. Rev. B 102 (2020) 165151. doi:10.1103/PhysRevB.102.165151. URL https://link.aps.org/doi/10.1103/PhysRevB.102.165151

[61] I. A. Ado, I. A. Dmitriev, P. M. Ostrovsky, M. Titov, Europhysics Letters 111 (3) (2015) 37004. doi:10.1209/0295$5075 / 111 / 37004$.

[62] E. J. König, P. M. Ostrovsky, M. Dzero, A. Levchenko, Anomalous hall effect on the surface of topological kondo insulators, Phys. Rev. B 94 (2016) 041403. doi:10.1103/PhysRevB.94.041403. URL https://link.aps.org/doi/10.1103/PhysRevB.94.041403

[63] E. J. König, A. Levchenko, Kerr effect from diffractive skew scattering in chiral $p_{x} \pm i p_{y}$ superconductors, Phys. Rev. Lett. 118 (2017) 027001. doi:10.1103/PhysRevLett.118.027001. 
URL https://link.aps.org/doi/10.1103/PhysRevLett.118.027001

[64] M. Milletarì, A. Ferreira, Quantum diagrammatic theory of the extrinsic spin hall effect in graphene, Phys. Rev. B 94 (2016) 134202. doi:10.1103/PhysRevB.94.134202. URL https://link.aps.org/doi/10.1103/PhysRevB.94.134202

[65] I. A. Ado, I. A. Dmitriev, P. M. Ostrovsky, M. Titov, Anomalous hall effect in a 2d rashba ferromagnet, Phys. Rev. Lett. 117 (2016) 046601. doi:10.1103/PhysRevLett.117.046601. URL https://link.aps.org/doi/10.1103/PhysRevLett.117.046601

[66] I. A. Ado, I. A. Dmitriev, P. M. Ostrovsky, M. Titov, Sensitivity of the anomalous hall effect to disorder correlations, Phys. Rev. B 96 (2017) 235148. doi:10.1103/PhysRevB.96.235148. URL https://link.aps.org/doi/10.1103/PhysRevB.96.235148

[67] E. Deyo, L. Golub, E. Ivchenko, B. Spivak, Semiclassical theory of the photogalvanic effect in non-centrosymmetric systems, arXiv preprint arXiv:0904.1917.

[68] J. E. Sipe, A. I. Shkrebtii, Second-order optical response in semiconductors, Physical Review B 61 (8) (2000) $5337-5352$.

[69] L. Wu, S. Patankar, T. Morimoto, N. L. Nair, E. Thewalt, A. Little, J. G. Analytis, J. E. Moore, J. Orenstein, Giant anisotropic nonlinear optical response in transition metal monopnictide weyl semimetals, Nature Physics 13 (4) (2017) 350-355. doi:10.1038/nphys3969. URL https://doi.org/10.1038/nphys3969

[70] Q. Ma, S.-Y. Xu, C.-K. Chan, C.-L. Zhang, G. Chang, Y. Lin, W. Xie, T. Palacios, H. Lin, S. Jia, P. A. Lee, P. JarilloHerrero, N. Gedik, Direct optical detection of weyl fermion chirality in a topological semimetal, Nature Physics 13 (9) (2017) 842-847. doi:10.1038/nphys4146.

URL https://doi.org/10.1038/nphys4146

[71] F. de Juan, A. G. Grushin, T. Morimoto, J. E. Moore, Quantized circular photogalvanic effect in weyl semimetals, Nature Communications 8 (1) (2017) $1-7$.

[72] T. Morimoto, S. Zhong, J. Orenstein, J. E. Moore, Semiclassical theory of nonlinear magneto-optical responses with applications to topological dirac/weyl semimetals, Phys. Rev. B 94 (2016) 245121. doi:10.1103/PhysRevB.94.245121. URL https://link.aps.org/doi/10.1103/PhysRevB.94.245121

[73] E. J. König, H.-Y. Xie, D. A. Pesin, A. Levchenko, Photogalvanic effect in weyl semimetals, Phys. Rev. B 96 (2017) 075123. doi:10.1103/PhysRevB.96.075123. URL https://link.aps.org/doi/10.1103/PhysRevB.96.075123

[74] L. E. Golub, E. L. Ivchenko, Circular and magnetoinduced photocurrents in weyl semimetals, Phys. Rev. B 98 (2018) 075305. doi:10.1103/PhysRevB.98.075305.

URL https://link.aps.org/doi/10.1103/PhysRevB.98.075305

[75] I. Sodemann, L. Fu, Quantum nonlinear hall effect induced by berry curvature dipole in time-reversal invariant materials, Physical Review Letters 115 (21) (2015) 216806.

[76] S. Singh, J. Kim, K. M. Rabe, D. Vanderbilt, Engineering weyl phases and nonlinear hall effects in $\mathrm{t}_{d}$-mote 2 , Phys. Rev. Lett. 125 (2020) 046402. doi:10.1103/PhysRevLett.125.046402. URL https://link.aps.org/doi/10.1103/PhysRevLett.125.046402

[77] E. J. König, M. Dzero, A. Levchenko, D. A. Pesin, Gyrotropic hall effect in berry-curved materials, Phys. Rev. B 99 (2019) 155404. doi:10.1103/PhysRevB.99.155404. URL https://link.aps.org/doi/10.1103/PhysRevB.99.155404

[78] H. Isobe, S.-Y. Xu, L. Fu, High-frequency rectification via chiral bloch electrons, Science Advances 6 (13). doi:10.1126/sciadv.aay2497.

[79] Z. Z. Du, C. M. Wang, S. Li, H.-Z. Lu, X. C. Xie, Disorder-induced nonlinear hall effect with time-reversal symmetry, Nature Communications 10 (1) (2019) 3047. doi:10.1038/s41467-019-10941-3. URL https://doi.org/10.1038/s41467-019-10941-3

[80] S. Nandy, I. Sodemann, Symmetry and quantum kinetics of the nonlinear hall effect, Phys. Rev. B 100 (2019) 195117. doi:10.1103/PhysRevB.100.195117. URL https://link.aps.org/doi/10.1103/PhysRevB.100.195117

[81] C. Xiao, Z. Z. Du, Q. Niu, Theory of nonlinear hall effects: Modified semiclassics from quantum kinetics, Phys. Rev. B 100 (2019) 165422. doi:10.1103/PhysRevB.100.165422. URL https://link.aps.org/doi/10.1103/PhysRevB.100.165422

[82] J. L. Mañes, Existence of bulk chiral fermions and crystal symmetry, Phys. Rev. B 85 (2012) 155118. doi:10.1103/PhysRevB.85.155118. URL https://link.aps.org/doi/10.1103/PhysRevB.85.155118

[83] B. J. Wieder, Y. Kim, A. M. Rappe, C. L. Kane, Double dirac semimetals in three dimensions, Phys. Rev. Lett. 116 (2016) 186402. doi:10.1103/PhysRevLett.116.186402. URL https://link.aps.org/doi/10.1103/PhysRevLett.116.186402

[84] B. Bradlyn, J. Cano, Z. Wang, M. Vergniory, C. Felser, R. Cava, B. A. Bernevig, Beyond dirac and weyl fermions: Unconventional quasiparticles in conventional crystals, Science 353 (6299).

[85] A. Bouhon, A. M. Black-Schaffer, Global band topology of simple and double dirac-point semimetals, Phys. Rev. B 95 (2017) 241101. doi:10.1103/PhysRevB.95.241101. URL https://link.aps.org/doi/10.1103/PhysRevB.95.241101

[86] P. Tang, Q. Zhou, S.-C. Zhang, Multiple types of topological fermions in transition metal silicides, Physical review letters 119 (20) (2017) 206402.

[87] N. B. Schröter, D. Pei, M. G. Vergniory, Y. Sun, K. Manna, F. De Juan, J. A. Krieger, V. Süss, M. Schmidt, P. Dudin, 
et al., Chiral topological semimetal with multifold band crossings and long fermi arcs, Nature Physics 15 (8) (2019) $759-765$.

[88] F. Flicker, F. de Juan, B. Bradlyn, T. Morimoto, M. G. Vergniory, A. G. Grushin, Chiral optical response of multifold fermions, Phys. Rev. B 98 (2018) 155145. doi:10.1103/PhysRevB.98.155145.

URL https://link.aps.org/doi/10.1103/PhysRevB.98.155145

[89] M.-A. Sánchez-Martínez, F. de Juan, A. G. Grushin, Linear optical conductivity of chiral multifold fermions, Phys. Rev. B 99 (2019) 155145. doi:10.1103/PhysRevB.99.155145.

URL https://link.aps.org/doi/10.1103/PhysRevB.99.155145

[90] T. Habe, Dynamical conductivity in the multiply degenerate point-nodal semimetal cosi, Phys. Rev. B 100 (2019) 245131. doi:10.1103/PhysRevB.100.245131. URL https://link.aps.org/doi/10.1103/PhysRevB.100.245131

[91] S. Nandy, S. Manna, D. Călugăru, B. Roy, Generalized triple-component fermions: Lattice model, fermi arcs, and anomalous transport, Physical Review B 100 (23) (2019) 235201.

[92] B. Sadhukhan, T. Nag, The role of time reversal symmetry and tilting in circular photogalvanic responses, arXiv preprint arXiv:2009.14333.

[93] L. Z. Maulana, K. Manna, E. Uykur, C. Felser, M. Dressel, A. V. Pronin, Optical conductivity of multifold fermions: The case of rhsi, Phys. Rev. Research 2 (2020) 023018. doi:10.1103/PhysRevResearch.2.023018. URL https://link.aps.org/doi/10.1103/PhysRevResearch.2.023018

[94] G. Chang, S.-Y. Xu, B. J. Wieder, D. S. Sanchez, S.-M. Huang, I. Belopolski, T.-R. Chang, S. Zhang, A. Bansil, H. Lin, M. Z. Hasan, Unconventional chiral fermions and large topological fermi arcs in rhsi, Phys. Rev. Lett. 119 (2017) 206401. doi:10.1103/PhysRevLett.119.206401. URL https://link.aps.org/doi/10.1103/PhysRevLett.119.206401

[95] D. Rees, K. Manna, B. Lu, T. Morimoto, H. Borrmann, C. Felser, J. E. Moore, D. H. Torchinsky, J. Orenstein, Helicitydependent photocurrents in the chiral weyl semimetal rhsi, Science Advances 6 (29). doi:10.1126/sciadv.aba0509.

[96] B. J. Wieder, B. Bradlyn, Z. Wang, J. Cano, Y. Kim, H.-S. D. Kim, A. M. Rappe, C. Kane, B. A. Bernevig, Wallpaper fermions and the nonsymmorphic dirac insulator, Science 361 (6399) (2018) 246-251.

[97] D. Bercioux, D. F. Urban, H. Grabert, W. Häusler, Massless dirac-weyl fermions in a $\sqcup_{3}$ optical lattice, Phys. Rev. A 80 (2009) 063603. doi:10.1103/PhysRevA.80.063603. URL https://link.aps.org/doi/10.1103/PhysRevA.80.063603

[98] D. Green, L. Santos, C. Chamon, Isolated flat bands and spin-1 conical bands in two-dimensional lattices, Phys. Rev. B 82 (2010) 075104. doi:10.1103/PhysRevB.82.075104. URL https://link.aps.org/doi/10.1103/PhysRevB.82.075104

[99] V. Apaja, M. Hyrkäs, M. Manninen, Flat bands, dirac cones, and atom dynamics in an optical lattice, Phys. Rev. A 82 (2010) 041402. doi:10.1103/PhysRevA.82.041402. URL https://link.aps.org/doi/10.1103/PhysRevA.82.041402

[100] R. Shen, L. B. Shao, B. Wang, D. Y. Xing, Single dirac cone with a flat band touching on line-centered-square optical lattices, Phys. Rev. B 81 (2010) 041410. doi:10.1103/PhysRevB.81.041410. URL https://link.aps.org/doi/10.1103/PhysRevB.81.041410

[101] K. Essafi, L. D. C. Jaubert, M. Udagawa, Flat bands and dirac cones in breathing lattices, Journal of Physics: Condensed Matter 29 (31) (2017) 315802. doi:10.1088/1361-648x/aa782f.

[102] Y.-Q. Zhu, D.-W. Zhang, H. Yan, D.-Y. Xing, S.-L. Zhu, Emergent pseudospin-1 maxwell fermions with a threefold degeneracy in optical lattices, Phys. Rev. A 96 (2017) 033634. doi:10.1103/PhysRevA.96.033634. URL https://link.aps.org/doi/10.1103/PhysRevA.96.033634

[103] L. Wang, D.-X. Yao, Coexistence of spin-1 fermion and dirac fermion on the triangular kagome lattice, Phys. Rev. B 98 (2018) 161403. doi:10.1103/PhysRevB.98.161403. URL https://link.aps.org/doi/10.1103/PhysRevB.98.161403

[104] E. J. König, P. Coleman, Crystalline-symmetry-protected helical majorana modes in the iron pnictides, Phys. Rev. Lett. 122 (2019) 207001. doi:10.1103/PhysRevLett.122.207001. URL https://link.aps.org/doi/10.1103/PhysRevLett.122.207001

[105] B. L. Altshuler, Sov. Phys. JETP 48 (4) (1978) 670-675.

[106] A. Kamenev, Field Theory of Non-Equilibrium Systems, Cambridge University Press, 2011. URL https://books.google.de/books?id=CwlrUepnla4C

[107] D. A. Pesin, Two-particle collisional coordinate shifts and hydrodynamic anomalous hall effect in systems without lorentz invariance, Phys. Rev. Lett. 121 (2018) 226601. doi:10.1103/PhysRevLett.121.226601. URL https://link.aps.org/doi/10.1103/PhysRevLett.121.226601

[108] C. Xiao, Y. Liu, M. Xie, S. A. Yang, Q. Niu, Theory of the phonon side-jump contribution in anomalous hall effect, Phys. Rev. B 99 (2019) 245418. doi:10.1103/PhysRevB.99.245418. URL https://link.aps.org/doi/10.1103/PhysRevB.99.245418 